\title{
Simulation of an Evolving Convective Boundary Layer Using a Scale-Dependent Dynamic Smagorinsky Model at Near-Gray-Zone Resolutions
}

\author{
G. A. Efstathiou \\ Department of Mathematics, Centre for Geophysical and Astrophysical Fluid Dynamics, \\ University of Exeter, Exeter, United Kingdom \\ R. S. PLANT \\ Department of Meteorology, University of Reading, Reading, United Kingdom
}

M.-J. M. BOPAPE

South African Weather Service, Pretoria, South Africa

(Manuscript received 2 November 2017, in final form 10 July 2018)

\begin{abstract}
A scale-dependent Lagrangian-averaged dynamic Smagorinsky subgrid scheme with stratification effects is used to simulate the evolving convective boundary layer of the Wangara (Australia) case study in the grayzone regime (specifically, for grid lengths from 25 to $400 \mathrm{~m}$ ). The dynamic Smagorinsky and standard Smagorinsky approaches are assessed for first- and second-order quantities in comparison with results derived from coarse-grained large-eddy simulation (LES) fields. In the LES regime, the subgrid schemes produce very similar results, albeit with some modest differences near the surface. At coarser resolutions, the use of the standard Smagorinsky approach significantly delays the onset of resolved turbulence, with the delay increasing with coarsening resolution. In contrast, the dynamic Smagorinsky scheme much improves the spinup and so is also able to maintain consistency with the LES temperature profiles at the coarser resolutions. Moreover, the resolved part of the turbulence reproduces well the turbulence profiles obtained from the coarse-grained fields, especially in the near gray zone. The dynamic scheme does become somewhat overenergetic with further coarsening of the resolution, especially near the surface. The dynamic scheme reaches its limit in the current configuration when the test filter starts to sample at the unresolved scales, returning very small Smagorinsky coefficients. Sensitivity tests reveal that the dynamic model can adapt to changes in the imposed numerical or subgrid diffusion by adjusting the Smagorinsky constant to the changing flow field and minimizing the dissipation effects on the resolved turbulence structures.
\end{abstract}

\section{Introduction}

Large-eddy simulation (LES) is a technique where the energy-containing turbulent structures are resolved while the smaller-scale isotropic turbulence is parameterized. The separation of scales can be achieved by spatially filtering the Navier-Stokes equations at the filter scale $\Delta$. The subfilter (or subgrid) motions are commonly represented using an eddy-viscosity approach based on a Smagorinsky or a turbulent kinetic energy (TKE) closure. The Smagorinsky model (Smagorinsky 1963; Lilly 1967) aims to remove energy from the

\footnotetext{
Corresponding author: G. A. Efstathiou, g.efstathiou@exeter. ac.uk
}

production to the dissipation scales based on the mixing length hypothesis [see Eqs. (3) and (4)] with the mixing length taken as the product of the gridscale filter $\Delta$ and the Smagorinsky constant $C_{S}$. In principle, the Smagorinsky approach is valid when $\Delta$ lies within the inertial subrange. However, approaching the lower boundary (wall or surface) the grid scale perforce approaches the dominant turbulence length scales, and therefore much of the TKE production is transferred to the unresolved scales. Specifically, in the vicinity of the lower boundary the Smagorinsky scheme has been found to become too dissipative, and as a result a transition function is necessary (see Mason and Thomson 1992) to represent the impact of the lower boundary on the turbulence length scales. 
To overcome the limitations of using a constant $C_{S}$, Germano et al. (1991) developed a dynamic procedure enabling the calculation of $C_{S}$ as a function of the resolved flow. Making use of the Germano identity, the key concept of the dynamic procedure is to sample the smallest resolvable scales by introducing a test filter, usually chosen to be 2 times the gridscale filter. The approach assumes a scale invariance in the form $C_{S}(\Delta)=C_{S}(2 \Delta)$ and so requires $\Delta$ to lie within the inertial subrange. Because scale invariance is not guaranteed when the grid scale and the dominant turbulence length scales are not well separated, Porté-Agel et al. (2000) developed a scaledependent extension to the dynamic model by applying a second test-filter operation, which allows for a determination of the variation of $C_{S}$ across the test scales. The scale-dependent model exhibited improved dissipation characteristics and mean velocity profiles compared to Smagorinsky and Germano dynamic schemes. Meneveau et al. (1996) proposed a Lagrangian timeaveraging formulation suitable for simulations over nonhomogeneous surface roughness. Bou-Zeid et al. (2005) introduced the scale dependence of $C_{S}$ in the Lagrangian model of Meneveau et al. (1996) using two test filters, similar to work done by Porté-Agel et al. (2000).

The horizontal resolution of numerical weather prediction (NWP) models has continued to increase in recent years, and models are increasingly becoming able to operate at grid resolutions that are comparable to the dominant turbulence production scales. Such resolutions constitute the gray zone (Wyngaard 2004; Beare 2014) for boundary layer $(\mathrm{BL})$ processes where neither strict LES nor Reynolds-averaged Navier-Stokes (RANS) formulations can fully represent the turbulent transports in the BL. Beare (2014) defined a dissipation length to identify the onset of the gray zone and demonstrated that the gray zone is manifested at grid spacings smaller than the dominant turbulence length scales. At gray-zone resolutions, the dominant turbulent structures become partially resolved and are sensitive to the choice of subgrid mixing (e.g., Honnert et al. 2011; Efstathiou and Beare 2015; Shin and Hong 2015). For example, the choice of the turbulent mixing length if using the standard Smagorinsky approach controls the partitioning between resolved and parameterized TKE (Efstathiou and Beare 2015; Efstathiou et al. 2016) and controls cumulus cloud morphologies (Hanley et al. 2015; Stein et al. 2015). On the other hand, one-dimensional (1D) ensemble-mean mesoscale parameterizations seem to remove too much energy from the resolved scales (Honnert et al. 2011; Shin and Hong 2013, 2015). Partly for this reason, some studies have experimented with methods for adding stochastic variability to the boundary layer in gray-zone NWP simulations based on mesoscale BL parameterizations
(Leoncini et al. 2013; Kober and Craig 2016). Moreover, Shin and Dudhia (2016) examined five mesoscale BL parameterizations in the gray zone and concluded that they are not scale aware, each performing best at certain resolutions.

A number of approaches have been developed for the parameterization of subgrid processes at gray-zone resolutions. Honnert et al. (2011) introduced a new similarity relationship to describe the partitioning between resolved and subfilter scales as a function of scale. This has been used by Boutle et al. (2014) to blend between a Smagorinsky parameterization and a 1D BL parameterization (Lock et al. 2000). It has also been used to modify a mass-flux scheme (Honnert et al. 2016). Along similar lines, Shin and Hong (2015) developed a parameterization based on a nonlocal K-profile scheme (Hong et al. 2006) while Ito et al. (2015) adjusted the dissipation length scales in the TKE Mellor-Yamada scheme to account for gray-zone mixing. Additionally, Bhattacharya and Stevens (2016) developed a twoequation TKE model to describe turbulent transports partitioned into LES and RANS frameworks.

Efstathiou and Beare (2015) modified the standard Smagorinsky scheme with a bounding approach for the diffusion in an effort to preserve the inherent diffusivity of the flow across scales. Efstathiou et al. (2016) compared the bounding and blending approaches in simulations of the morning development of a convective BL (CBL) for two case studies at gray-zone resolutions with the Met Office Large Eddy Model. It was shown that each approach has particular strengths and weaknesses, with blending performing better when $\mathrm{BL}$ is shallow whereas bounding improves both spinup and the deepBL representation. As shown by Zhou et al. (2014), who also studied the CBL for the Wangara (Australia) case study, turbulence initiation in the gray zone is dependent on horizontal grid spacing.

In this study, the Lagrangian-averaged scaledependent dynamic Smagorinsky model (LASD) as presented in Bou-Zeid et al. (2005) is modified to account for stratification effects and used to simulate the evolving Wangara $\mathrm{CBL}$ at different resolutions. A number of previous studies have used different versions of dynamic Smagorinsky models to simulate atmospheric flows (e.g., Kumar et al. 2006; Huang et al. 2008; Stoll and Porté-Agel 2008) and demonstrated benefits from a dynamically calculated $C_{S}$ at LES resolutions. Basu et al. (2008) used a Lagrangian scale-dependent subgrid model in a pseudospectral code to simulate the full diurnal cycle of the Wangara case study. They showed that the dynamic Smagorinsky model was able to reproduce the characteristics of the CBL and the nighttime stable BL even at a relatively coarse LES grid length of $62.5 \mathrm{~m}$. 
Here, we extend LASD simulations of the case up to $400 \mathrm{~m}$ horizontal grid length ( $\Delta x=\Delta y$ in this study) to examine the behavior of the scheme well beyond the LES regime. Our aim is to establish whether LASD continues to provide benefits for atmospheric modeling into the gray-zone regime and hence whether it may provide a good alternative to the current use of the standard Smagorinsky scheme in various NWP applications (e.g., Hanley et al. 2015; Thurston et al. 2016). During the morning development of the CBL, a grid length of $\Delta x=$ $400 \mathrm{~m}$ corresponds to $\Delta x / z_{i} \simeq 1-4$ (where $z_{i}$ is the BL depth), as compared with $\Delta x / z_{i} \simeq 0.15-0.65$ in the coarsest simulations considered by Basu et al. (2008). Even though a comparison between pseudospectral and finite-difference models is not straightforward, because pseudospectral codes tend to be more accurate, especially near the cutoff filter (see Glendening and Haack 2001), the simulations performed in this study clearly push the limits of the LASD usage.

According to the dissipation length analysis of Efstathiou et al. (2016), simulations with $\Delta x \gtrsim 100 \mathrm{~m}$ will lie within the gray zone (see their Fig. 11) when the BL is shallow for this case. Following the rapid BL development and the establishment of a sufficiently deep BL in the afternoon, these simulations are not strictly found in the gray zone. However, neither can they be considered LES converging according to Sullivan and Patton (2011). We therefore define this resolution regime as the near gray zone where more TKE is resolved than subgrid, even though TKE is still significantly underresolved relative to the LES. Our LASD results are compared with those from standard Smagorinsky runs and are validated against firstand second-order quantities derived from the coarse-grained fields of a converging LES run.

\section{The Large Eddy Model}

The Met Office Large Eddy Model (LEM), version 2.4, was used for the simulations conducted in this study. The Navier-Stokes equations with the Boussinesq approximation are solved in three dimensions using a centereddifference advection scheme (Piacsek and Williams 1970) for the momentum and a total variation diminishing scheme (TVD; Leonard et al. 1993) for the perturbation potential temperature equation. Two models are considered to account for the subgrid fluxes of heat and momentum.

\section{a. The Smagorinsky scheme}

An accurate representation of subgrid motions that are responsible for the transfer of energy from the resolved to the dissipation scales is needed to ensure that the turbulent structures are realistically resolved and not damped because of excessive mixing or appearing noisy because of insufficient diffusion. Smagorinsky (1963) introduced a local eddy-viscosity approach to model the subgrid stress tensor $\tau_{i j}$ :

$$
\tau_{i j}=-2 v_{t} \bar{S}_{i j}
$$

where

$$
\bar{S}_{i j}=\frac{1}{2}\left(\frac{\partial \bar{u}_{i}}{\partial x_{j}}+\frac{\partial \bar{u}_{j}}{\partial x_{i}}\right) .
$$

The overbar denotes resolved quantities, that is, filtered at the scale $\Delta$. The eddy viscosity $v_{t}$ is given by

$$
v_{t}=\lambda^{2}|\bar{S}|
$$

with $|\bar{S}|=\left(2 \bar{S}_{i j} \bar{S}_{i j}\right)^{1 / 2}$ and $\lambda$ being the mixing length:

$$
\lambda=C_{S} \Delta .
$$

The dimensionless quantity $C_{S}$ is known as the Smagorinsky constant.

The LEM, as for other large-eddy codes for atmospheric flows, takes account of stratification effects in subgrid turbulent transfer through the use of stability functions. Thus, the stress tensor becomes

$$
\tau_{i j}=-2 v_{t} \bar{S}_{i j} f_{m}(\mathrm{Ri}),
$$

where $f_{m}(\mathrm{Ri})$ is the stability function for momentum, which is dependent on the Richardson number $\mathrm{Ri}$.

Near the ground the turbulence length scales are limited by the presence of the solid boundary, and hence $\Delta$ becomes comparable to the production scales and the model becomes overdissipative. To avoid excessive dissipation close to the ground, a wall-damping function for $\lambda$ was introduced by Mason and Thomson (1992):

$$
\frac{1}{\lambda^{2}}=\frac{1}{(k z)^{2}}+\frac{1}{\left(C_{S} \Delta\right)^{2}}
$$

The default LEM configuration uses $C_{S}=0.23$ and $\Delta=\Delta x$. Similarly, for the subgrid heat flux $q_{i}$ we have

$$
q_{i}=-\frac{v_{t}}{\operatorname{Pr}} \frac{\partial \bar{\theta}}{\partial x_{i}} f_{h}(\mathrm{Ri})
$$

where Pr is the Prandtl number equal to 0.7 and $f_{h}(\mathrm{Ri})$ is the stability function for heat.

\section{b. Lagrangian-averaged scale-dependent dynamic Smagorinsky model}

The dynamic procedure aims at calculating an appropriate $C_{S}$ value for the subgrid fluxes based on the 
smallest resolved scales in the simulation. Our approach follows the implementation of Bou-Zeid et al. (2005). However, it also includes the effects of stratification by incorporating the stability functions as a self-consistent aspect of the dynamic procedure. The scale-dependent procedure is based on applying the same subgrid model (i.e., Smagorinsky) at the grid scale and at two different test filter scales. The Germano identity (Germano et al. 1991) relates the subgrid stresses at different scales:

$$
L_{i j}=T_{i j}-\widetilde{\tau_{i j}}=\widetilde{\bar{u}}_{i} \bar{u}_{j}-\widetilde{\bar{u}}_{i} \widetilde{\bar{u}}_{j},
$$

where the tilde denotes test filtering at the scale $2 \Delta, T_{i j}$ is the subgrid stress tensor at scale $2 \Delta$, and $L_{i j}$ is the resolved stress tensor that expresses length scales intermediate between $\Delta$ and $2 \Delta$. Note that $\tau_{i j}, T_{i j}$, and $L_{i j}$ here denote the deviatoric part of the tensor.

Applying the Smagorinsky model [Eqs. (3)-(5)] at the test filter scale results in

$$
T_{i j}=-2 C_{S_{2 \Delta}}^{2}(2 \Delta)^{2}|\widetilde{\bar{S}}| \tilde{\bar{S}}_{i j} \tilde{f}_{m}(\mathrm{Ri}) .
$$

Substituting Eqs. (5) and (9) into Eq. (8) results in an equation describing the error from the use of the Smagorinsky model in Eq. (8):

$$
e_{i j}=L_{i j}-C_{S_{\Delta}}^{2} M_{i j}
$$

with

$$
M_{i j}=2 \Delta^{2}\left[\overline{|\bar{S}| \bar{S}_{i j} f_{m}(\mathrm{Ri})}-4 \beta|\widetilde{\bar{S}}| \widetilde{\bar{S}}_{i j} \tilde{f}_{m}(\mathrm{Ri})\right] .
$$

Here the $2 \Delta$ filtering operation in the first term in square brackets extends over all three factors forming the product. The introduced parameter $\beta$ is defined as

$$
\beta=\frac{C_{S_{2 \Delta}}}{C_{S_{\Delta}}}
$$

and denotes the scale dependence of the $C_{S}$ parameter.

Scale invariance corresponds to the choice of $\beta=1$. Lilly (1992) proposed a least squares approach for the minimization of Eq. (10), and this results in the following form:

$$
C_{S_{\Delta}}^{2}=C_{S_{2 \Delta}}^{2}=\frac{\left\langle L_{i j} M_{i j}\right\rangle}{\left\langle M_{i j} M_{i j}\right\rangle} .
$$

The indices are to be contracted. Angled brackets here indicate an averaging procedure, which is not a requirement of the dynamic approach, but an averaging of some description is normally performed in practice, not least because the Smagorinsky values would otherwise exhibit large variability in time and space. We return to this point later.
When $\Delta$ is comparable to the dominant production scales of turbulence, the scale invariance assumption must break down because the test-filter scale does not lie within the inertial subrange. In an attempt to overcome this problem and provide a dynamic gray-zone approach, a second testfilter scale can be introduced at $4 \Delta$ according to Porté-Agel et al. (2000; see also Bou-Zeid et al. 2005). The idea is that $\beta$ can then be estimated by making use of the information between the two test-filter scales. Applying the same dynamic procedure as above results in

$$
C_{S_{4 \Delta}}^{2}=\frac{\left\langle Q_{i j} N_{i j}\right\rangle}{\left\langle N_{i j} N_{i j}\right\rangle},
$$

where

$$
Q_{i j}=\widehat{\bar{u}}_{i} \widehat{\bar{u}}_{j}-\widehat{\bar{u}}_{i} \widehat{\bar{u}}_{j}
$$

and

$$
N_{i j}=2 \Delta^{2}\left[\overline{|\bar{S}| \bar{S}_{i j} f_{m}(\mathrm{Ri})}-16 \beta^{2}\left|\widehat{\bar{S}}_{\mid}\right| \hat{\bar{S}}_{i j} \hat{f}_{m}(\mathrm{Ri})\right],
$$

with the caret denoting filtering at the scale $4 \Delta$.

We assume now that $C_{S}$ is scale dependent but that the parameter $\beta$ is scale invariant. That is,

$$
\beta=\frac{C_{S_{4 \Delta}}}{C_{S_{2 \Delta}}}=\frac{C_{S_{2 \Delta}}}{C_{S_{\Delta}}},
$$

and therefore

$$
\beta^{2}=\frac{C_{S_{4 \Delta}}}{C_{S_{\Delta}}} .
$$

Thus, considering a spectral plot, the method attempts to preserve the slope of the spectrum at the scales of $4 \Delta$ and below. Evaluating the Smagorinsky coefficient at the two filter scales by means of Eqs. (13) and (14), the $\beta$ parameter is calculated in practice by taking (see also Bou-Zeid et al. 2005)

$$
\beta=\max \left(\frac{C_{S_{4 \Delta}}}{C_{S_{2 \Delta}}}, 0.125\right),
$$

and then the Smagorinsky coefficient used at the grid scale is obtained from

$$
C_{S} \equiv C_{S_{\Delta}}=\frac{C_{S_{2 \Delta}}}{\beta}
$$

Note that the calculation of $\beta$ as proposed by Bou-Zeid et al. (2005) and presented in Eq. (19) is an approximation. For a more accurate prediction of the $C_{S}$ scale 
dependency, one should solve a fifth-order polynomial expression for $\beta$ (see Porté-Agel et al. 2000; Stoll and Porté-Agel 2008; Basu et al. 2008).

Regardless of whether one or two filter scales are in use (i.e., a scale-invariant or a scale-dependent $C_{S}$ ), the calculated $C_{S_{\Delta}}$ value is used in Eq. (5) instead of taking a constant value. Also the mixing length is calculated from Eq. (4) without imposing the wall-damping function [as in Eq. (6)]. This is because the mixing length is expected to be reduced in a realistic way toward the wall by means of the calculated $C_{S_{\Delta}}$, which is free to vary with height.

\section{1) LAGRANGIAN AVERAGING}

It was noted earlier that averaging is applied in dynamic model calculations. The approach of Germano et al. (1991) and Lilly (1992) was to use plane averaging, thereby assuming the flow to be horizontally homogeneous. Considering the scale-dependent dynamic models, Porté-Agel et al. (2000) also used plane averaging, whereas Bou-Zeid et al. (2005) and Stoll and Porté-Agel (2008) used Lagrangian averaging. Moreover, Basu and Porté-Agel (2006) formulated a local-averaging approach in which model coefficients are obtained dynamically by averaging locally on the horizontal plane with a stencil of $3 \times 3$ grid points (see also Basu et al. 2008).

The use of Lagrangian averaging should be more appropriate than plane averaging for inhomogeneous flows and allows for local horizontal variations in the mixing to be captured. The idea is discussed in Meneveau et al. (1996), and it defines a Lagrangian averaging operation for some test quantity $A(\mathbf{x}, t)$ along the fluid-particle trajectories according to

$$
\langle A(\mathbf{x}, t)\rangle=\int_{-\infty}^{t} A\left[\mathbf{x}\left(t^{\prime}\right), t^{\prime}\right] W\left(t-t^{\prime}\right) d t^{\prime} .
$$

Here $\mathbf{x}\left(t^{\prime}\right)$ is the position along the trajectory at the earlier time $t^{\prime}$ and $W\left(t-t^{\prime}\right)$ is a weighting function. Further details about the averaging procedure can be found in Meneveau et al. (1996) and Bou-Zeid et al. (2005). A key idea is that the averaging time scale is not set in advance but may itself depend on the characteristics of the turbulence.

\section{2) Treatment of STABILITY FUnCTIONS}

For the LEM implementation, we treat the stability functions (Brown et al. 1994) as being an inherent part of the subgrid model and therefore they are included in the filtering process for the different test scales as part of the dynamic procedure (Kirkpatrick et al. 2006). The stability functions depend on the Richardson number that is appropriate for the flow filtered to the test scale in question, so that $\tilde{\mathrm{R}} \mathrm{i}=\operatorname{Ri}\left(\widetilde{\overline{u_{i}}}, \widetilde{\overline{\theta_{i}}}\right)$, for example, which is distinct from $\operatorname{Ri}\left(\overline{u_{i}}, \overline{\theta_{i}}\right)$. Specifically then,

$$
\widetilde{\mathrm{Ri}}=\frac{\frac{g}{\theta_{0}} \frac{\partial \widetilde{\bar{\theta}}}{\partial z}}{|\widetilde{S}|^{2}} .
$$

On the scale of the $2 \Delta$ filter, for example, the functional forms used are given by the following: For $\widetilde{\mathrm{Ri}}<0$,

$$
\tilde{f}_{m}(\mathrm{Ri})=(1-16 \widetilde{\mathrm{Ri}})^{0.5} ;
$$

for $0 \leq \widetilde{\mathrm{Ri}}<0.25$,

$$
\tilde{f}_{m}(\mathrm{Ri})=\left(1-\frac{\widetilde{\mathrm{Ri}}}{0.25}\right)^{4}
$$

for $\widetilde{R i} \geq 0.25$,

$$
\tilde{f}_{m}(\mathrm{Ri})=0
$$

The same treatment of stability is followed at the grid scale with the tildes dropped and is also followed for the filtering at the $4 \Delta$ scale, with tildes replaced by carets in Eqs. (23)-(25) above.

\section{Simulations}

Two series of dry LEM simulations were performed of the daytime BL evolution of the Wangara day-33 case study (Clarke et al. 1971). One series uses the standard Smagorinsky (SMAG) scheme as specified in section $2 \mathrm{a}$, and the other uses the LASD scheme of section $2 \mathrm{~b}$. In each case, the horizontal resolution was varied from a value suitable for LES $(\Delta x=25 \mathrm{~m})$ through to values representative of the gray-zone regime (up to $\Delta x=400 \mathrm{~m}$ ). Our focus is on the ability of the two schemes to reproduce the basic characteristics of a typical, clear day BL at different resolutions.

The LEM setup follows the study of Efstathiou et al. (2016), starting from a shallow CBL of 100-m depth at 0900 LST and integrating for $9 \mathrm{~h}$. The vertical resolution was set to $\Delta z=0.4 \Delta x$ for the simulations with $\Delta x=25,50$, and $100 \mathrm{~m}$ and was kept as a constant $\Delta z=40 \mathrm{~m}$ for the coarser $\Delta x=200-$ and $400-\mathrm{m}$ simulations. A $40-\mathrm{m}$ vertical grid spacing is similar to that used within the boundary layer in operational high-resolution NWP models. The number of horizontal grid points used was $384 \times 384$ for the $25-\mathrm{m}$ simulations and reduced accordingly to maintain a domain size of $9.6 \times 9.6 \mathrm{~km}^{2}$ with no fewer than $48 \times 48$ grid points. The Prandtl number was not included in the dynamic calculations, and thus it was kept constant at its default value of 0.7 for our simulations, similar to Kumar et al. (2006).

For ease of reference, we will henceforth denote a simulation using the Smagorinsky scheme for a grid 

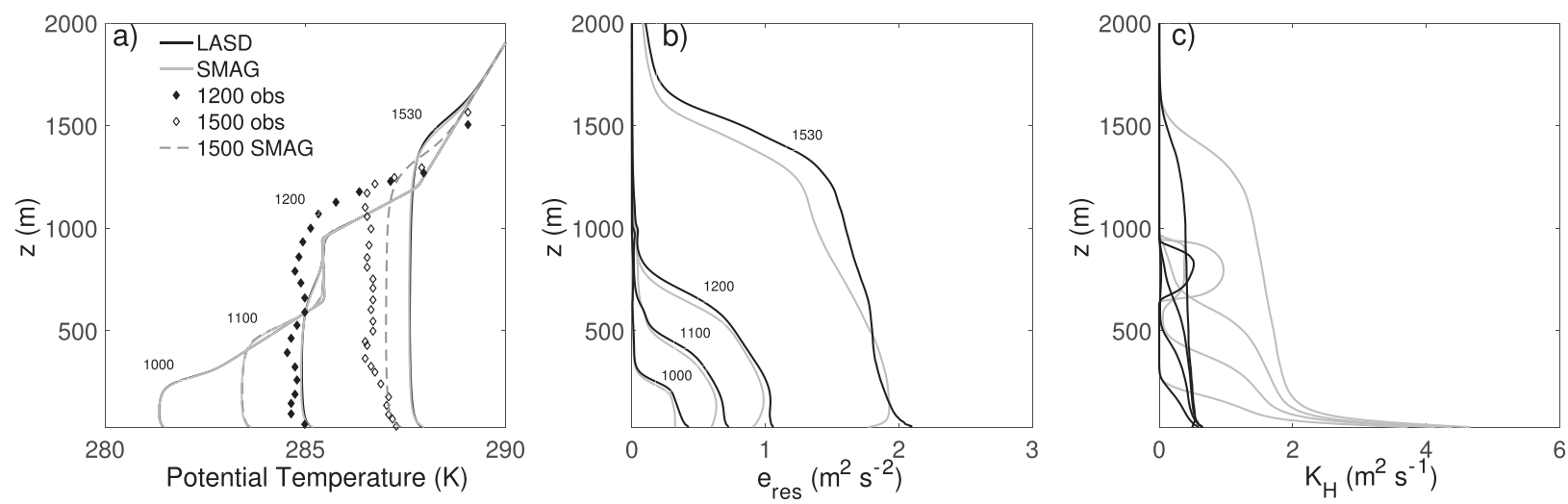

FIG. 1. Vertical profiles of horizontally averaged (a) potential temperature, (b) resolved TKE, and (c) eddy heat diffusion coefficient from the SMAG and LASD simulations with $\Delta x=25 \mathrm{~m}$. Results are shown for 1000, 1100, 1200, and 1530 LST during the CBL development. The filled and open diamonds represent potential temperature measurements at 1200 and 1500 LST, respectively, and the gray dashed line corresponds to the SMAG temperature profile at 1500 LST.

length of $\Delta x=25 \mathrm{~m}$ as the 25-m SMAG simulation and use analogous references for the simulations at other grid lengths and/or with the LASD subgrid scheme.

\section{Results: The LES regime}

The horizontally averaged potential temperature, resolved TKE, or $e_{\text {res }}$, and the eddy heat diffusion coefficient $K_{H}\left(=v_{t} / \mathrm{Pr}\right)$ are presented in Fig. 1 for the 25-m SMAG and LASD simulations. Results for four different times during the CBL development are shown. Additionally, the observed potential temperature profiles for 1200 and 1500 LST are included in Fig. 1, together with an extra SMAG profile for the latter time. The LES runs are in general able to capture the evolution of the observed temperature field at a similar level to Basu et al. (2008) and Nakanishi et al. (2014). Note that the purpose of this study is to examine the LASD behavior at coarse resolutions compared to the reference LES and not to validate the LEM against the observational Wangara dataset. The temperature profiles are almost identical for the SMAG and LASD schemes, although by 1530 LST the LASD produces a slightly deeper BL (see also the TKE profile in Fig. 1b). Similarly, the TKE profiles are very close, except near to the surface where the LASD simulation exhibits a maximum. The TKE differences in the surface layer, as well as near the inversion at 1530 LST, are mostly due to the increased resolved horizontal velocity variances $\left(\overline{u^{\prime 2}}\right.$ and $\left.\overline{v^{\prime 2}}\right)$ in the LASD run rather than $\overline{w^{\prime 2}}$, which does not differ significantly between the SMAG and LASD simulations (not shown).

It is known from previous studies that, in general, the dynamic model may fail to produce realistic momentum fluxes near the surface due to poorly resolved turbulence (Porté-Agel et al. 2000), and that the eddy diffusivity tends toward zero approaching the surface (Kirkpatrick et al. 2006). Nevertheless, the scale-dependent dynamic model has been shown to enhance dissipation near the surface, outperforming the traditional dynamic model (Bou-Zeid et al. 2005; Porté-Agel et al. 2000). PortéAgel et al. (2000) showed that dissipation derived from the scale-dependent model in the near-surface region was intermediate between that produced by the scaleinvariant dynamic model and that produced by the standard Smagorinsky. In any case, results from the 25-m LASD simulation exhibit less near-surface diffusion (Fig. 1c) relative to the SMAG simulation (as in Kirkil et al. 2012), although the resulting differences are not substantial, particularly for the first-order quantities. Because the 25-m SMAG simulation has been previously shown to lie within the LES convergent regime (Sullivan and Patton 2011; Efstathiou et al. 2016), it will be used as an LES reference in the following for examining the resolution sensitivity of the two schemes in an evolving CBL.

As noted above, Efstathiou et al. (2016) argued that simulations with $\Delta x \gtrsim 100 \mathrm{~m}$ would lie within the gray zone for the Wangara case study, and so we anticipate that the 50-m simulations should be similar to those with $\Delta x=25 \mathrm{~m}$ within the LES regime. Figure 2 shows the evolution of the averaged potential temperature and TKE profiles from the reference 25-m SMAG simulation in comparison with the 50-m SMAG and LASD simulations. No significant differences in the temperature profile are evident (except near the inversion because of the coarser vertical resolution in the 50-m simulations) during the CBL development. However, in examining the resolved TKE profiles in Fig. $2 b$, it can be seen that some differences emerge between the 50-m SMAG and LASD runs. LASD exhibits slightly stronger resolved TKE (closer to the reference simulation) compared to SMAG 

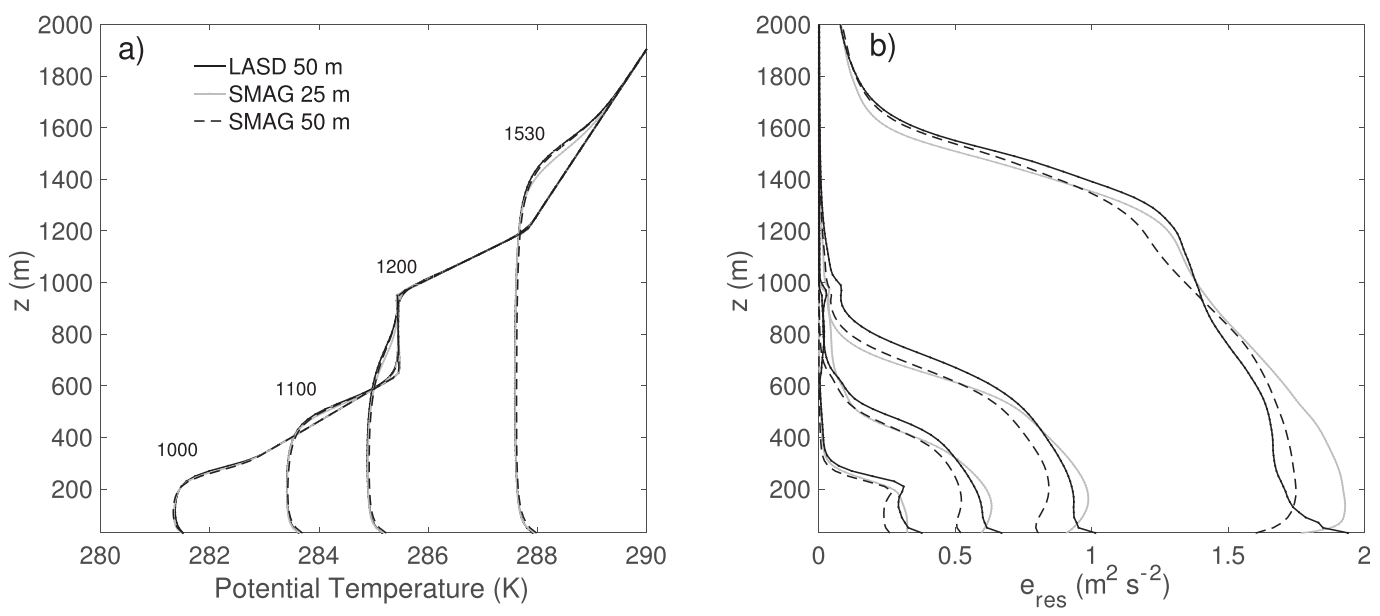

FIG. 2. Vertical profiles of horizontally averaged (a) potential temperature and (b) resolved TKE from the SMAG and LASD simulations with $\Delta x=50 \mathrm{~m}$ and from the SMAG simulation with $\Delta x=25 \mathrm{~m}$. Results are shown for 1000, 1100, 1200, and 1530 LST during the Wangara CBL development.

at the early stages of the BL development. By the time the BL has been fully developed (1530 LST), those differences have diminished in the bulk of the mixed layer whereas the reference run remains slightly more turbulent at the same time. It is also clear that, although LASD at $50 \mathrm{~m}$ produces some excess of resolved TKE near the ground, as discussed previously, this contrasts with a lack of near-surface resolved TKE in the 50-m SMAG simulation.

\section{Results: The gray-zone regime}

In Fig. 3, the vertical profiles of the averaged potential temperature from the SMAG and LASD simulations for different gray-zone resolutions are presented together with the 25-m reference SMAG simulation. Early in the simulations, SMAG produces superadiabatic profiles as illustrated at 1000 LST for the 100-m simulation, whereas for the 200- and 400-m simulations superadiabatic profiles persist until 1100 and 1300 LST, respectively. As the BL deepens, the SMAG potential temperature profiles converge toward the LES results and do not differ significantly among the runs. In contrast, the LASD profiles are well mixed at all times and for all three resolutions, reproducing well the LES results.

Figure 3 shows that the gray-zone SMAG simulations lack the necessary nonlocal mixing to neutralize the mixed layer when the BL is shallow (Zhou et al. 2014; Efstathiou et al. 2016). The reason for this is evident from examining the evolution of resolved TKE in the middle of the BL. The resolved TKE is shown in Fig. 4 for the various SMAG and LASD simulations and is compared with the TKE from the coarse-grained LES fields (Efstathiou and Beare 2015). The onset of resolved turbulence in the SMAG simulations is delayed
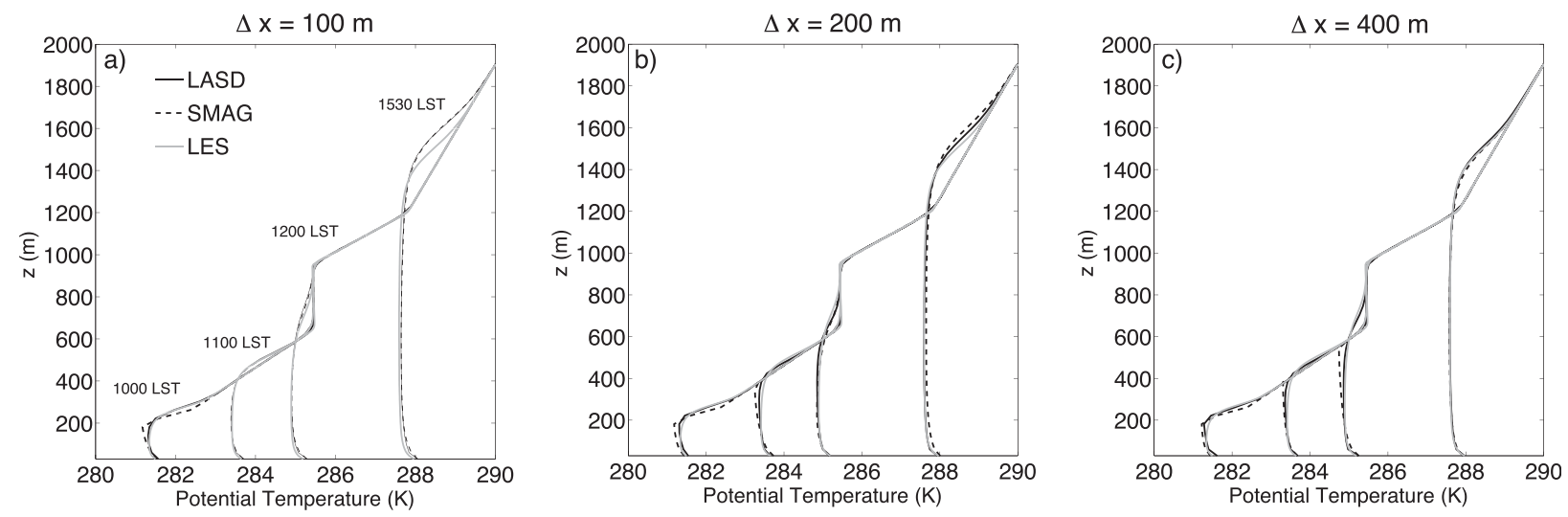

FIG. 3. Vertical profiles of horizontally averaged potential temperature from the SMAG and LASD simulations with $\Delta x=($ a) 100 , (b) 200, and (c) $400 \mathrm{~m}$ compared with the LES reference simulation (SMAG $25 \mathrm{~m}$ ). Results are shown for 1000, 1100, 1200, and 1530 LST during the Wangara CBL development. 


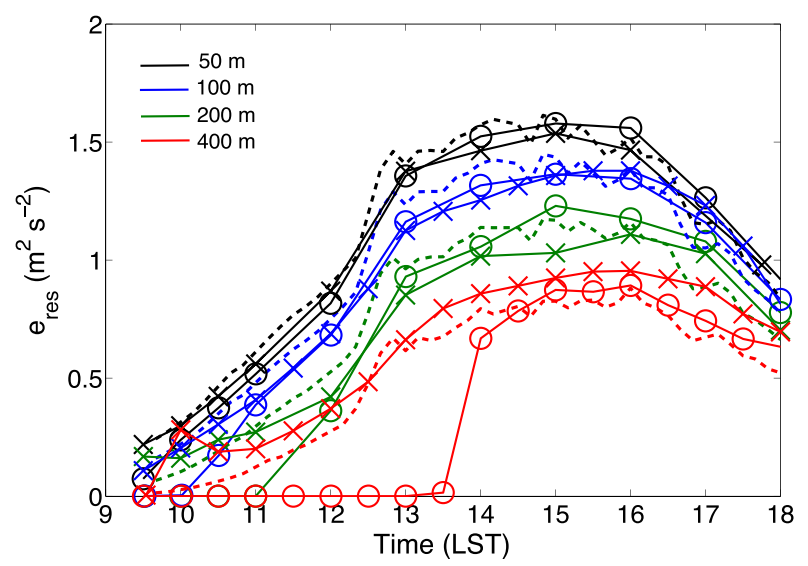

FIG. 4. Time series of horizontally averaged resolved TKE in the middle of the BL from the LASD (times signs) and SMAG (open circles) simulations for different grid lengths compared with the resolved TKE obtained after coarse-graining the LES fields into the same spatial scale (dashed lines). Results are shown for $\Delta x=50$ (black), 100 (blue), 200 (green), and 400 (red) m.

by around 1,2 , and $4 \mathrm{~h}$ for the 100-, 200-, and 400-m simulations, respectively. Once the gray-zone SMAG simulations do begin to resolve turbulence, then the temperature profiles become well mixed (Fig. 3) indicating the initiation of nonlocal thermal structures.

The TKE time series for the 50-m LASD and SMAG simulations agree very well with the filtered LES TKE, although the SMAG run is somewhat less energetic at the start of the simulation. The gray-zone LASD simulations do not exhibit any delay in the TKE initiation, and the TKE time series follow the series of the filtered fields. The 200-m LASD simulation is slightly overenergetic relative to the filtered TKE during the early stages of BL development at times when the corresponding SMAG simulation fails to resolve any overturning motions. Later in the afternoon, however, this LASD simulation is consistently less energetic compared to both SMAG and the filtered TKE. For the case of the 400-m simulation, LASD produces more turbulent fields than the coarsegrained results throughout the day and especially during the morning CBL development.

\section{a. Comparison with the coarse-grained turbulent fields}

Profiles of the resolved velocity variances and heat fluxes obtained from the SMAG and LASD simulations for $\Delta x=100,200$, and $400 \mathrm{~m}$ are presented for two different times in Figs. 5, 6, and 7, respectively. The profiles may be compared with the corresponding quantities derived after coarse-graining the reference LES fields to the scale $\Delta x$. The coarse-grained fields are obtained following Honnert et al. (2011) by spatially averaging the horizontal LES grids at the new grid scale. One hour into the 100-m SMAG simulation (1000 LST), resolved turbulence has not yet spun up, as shown in Figs. 5a-c, and consistent with Fig. 4. All of the turbulent heat flux is therefore required to occur by means of the subgrid scheme, leading perforce to the formation of superadiabatic temperature profiles through the depth of the CBL (Fig. 3a) as the only means through which to establish a positive heat flux via Eq. (7). In contrast, the LASD vertical velocity variance and heat flux profiles are very close to the $100-\mathrm{m}$ filtered results and only $\overline{u^{\prime 2}}$ is slightly stronger than for the filtered fields (Fig. 5c).

By 1530 LST, the CBL has deepened and only small differences are present between the resolved turbulence and the filtered fields (Figs. 5d-f). Nonetheless, the 100-m LASD simulation is better able to reproduce the filtered vertical profiles than is SMAG, especially in terms of the maximum of $\overline{w^{\prime 2}}$ and the heat flux in the surface layer.

In the 200-m simulations (Fig. 6) SMAG takes longer to spin up, and by 1100 LST there are still no resolved turbulent fluctuations. In contrast, the LASD simulation has well-developed resolved turbulent fluxes at this time and captures reasonably well the resolved vertical velocity variance (Fig. 6a) and heat flux (Fig. 6b) compared to the filtered fields. The horizontal velocity variance from LASD matches the variance from the coarsegrained fields in the middle of the BL but is too large near the ground and within the inversion layer (Fig. 6c). During the well-developed stage of the CBL (Figs. 6d-f) both simulations are similar and in reasonable agreement with the filtered results, albeit with SMAG somewhat overestimating the vertical velocity variance and LASD somewhat overestimating the horizontal velocity variance.

As shown in Fig. 4, the 400-m SMAG simulation delays the onset of resolved turbulence by almost $4 \mathrm{~h}$. This is corroborated by Fig. 7, which demonstrates that the simulation has no resolved velocity variances or heat fluxes at 1100 LST. Again, the corresponding LASD simulation has well-developed resolved turbulent fluxes. However, it does overestimate the intensity of the resolved turbulence (Figs. 4 and $7 \mathrm{a}-\mathrm{c}$ ). In the welldeveloped CBL at 1530 LST, both the SMAG and LASD 400-m simulations are overactive in comparison with the filtered results (Figs. 7d-f). LASD exhibits slightly stronger sensible heat fluxes relative to SMAG.

The form of the turbulence in the simulations is illustrated by Fig. 8, which shows horizontal cross sections of the vertical velocity in the middle of the BL at 1530 LST. At a first glance, the model simulations seem to be in reasonable agreement with the coarse-grained fields in the fully developed BL in accordance with the vertical velocity variance profiles presented in Figs. 5d, 6d, and 7d. 

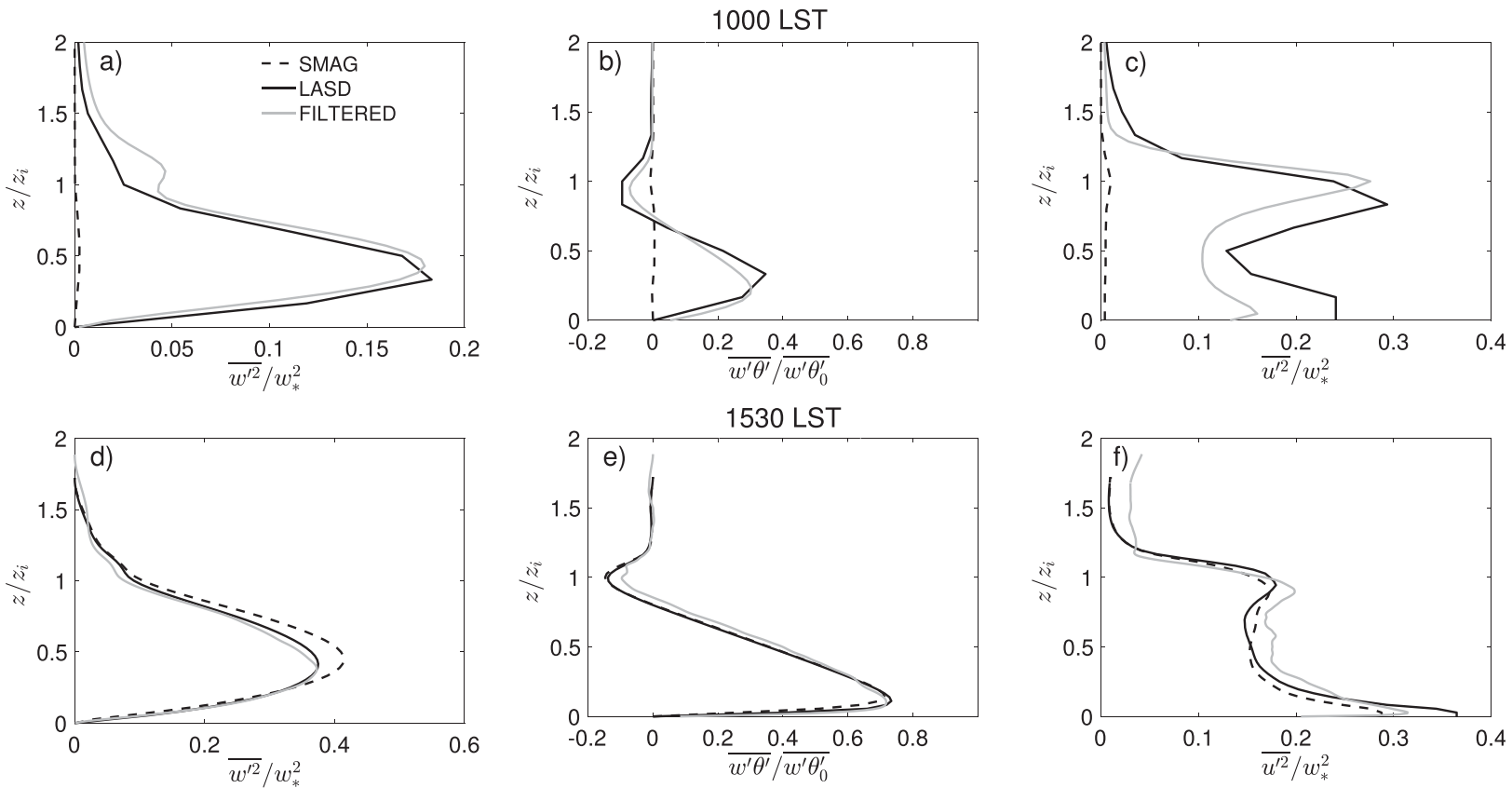

FIG. 5. Vertical profiles of horizontally averaged (a),(d) normalized resolved vertical velocity variance, (b),(e) normalized resolved sensible heat flux $\left.\overline{\left(w^{\prime} \theta^{\prime}\right.}\right)$, and (c),(f) normalized resolved horizontal velocity variance from the SMAG (dashed) and LASD (solid) 100-m simulations at (top) 1000 and (bottom) 1530 LST. Also shown (gray) are the profiles obtained from coarse-graining the reference LES fields to $100 \mathrm{~m}$. The BL depth $z_{i}$ is defined as the level of minimum heat flux and $w_{*}=\left[(g / \theta) \overline{z_{i}} \overline{w^{\prime} \theta_{0}^{\prime}}\right]^{1 / 3}$, with subscript 0 denoting the surface sensible heat flux.

However, on closer inspection, some interesting differences can be perceived. No significant differences are apparent between the SMAG and LASD plots for the 100-m simulations at this time. In comparison with the coarse-grained velocity field, however, both simulations exhibit slightly larger convective structures. The same remark applies more clearly in the $200-\mathrm{m}$ simulations, for which the structures also appear a little larger for SMAG
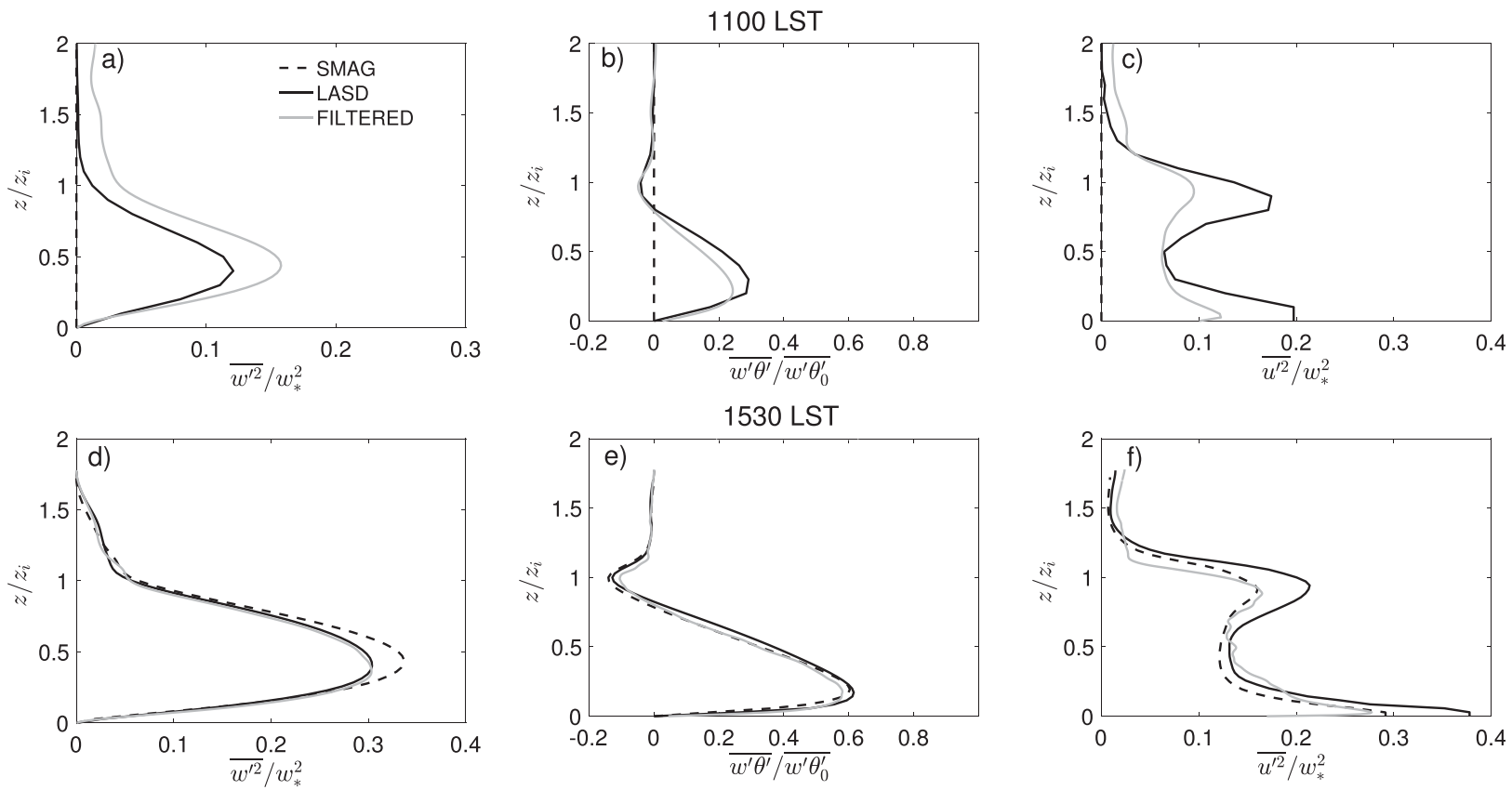

FIG. 6. As in Fig. 5, but for the 200-m simulations at 1100 and 1530 LST. 

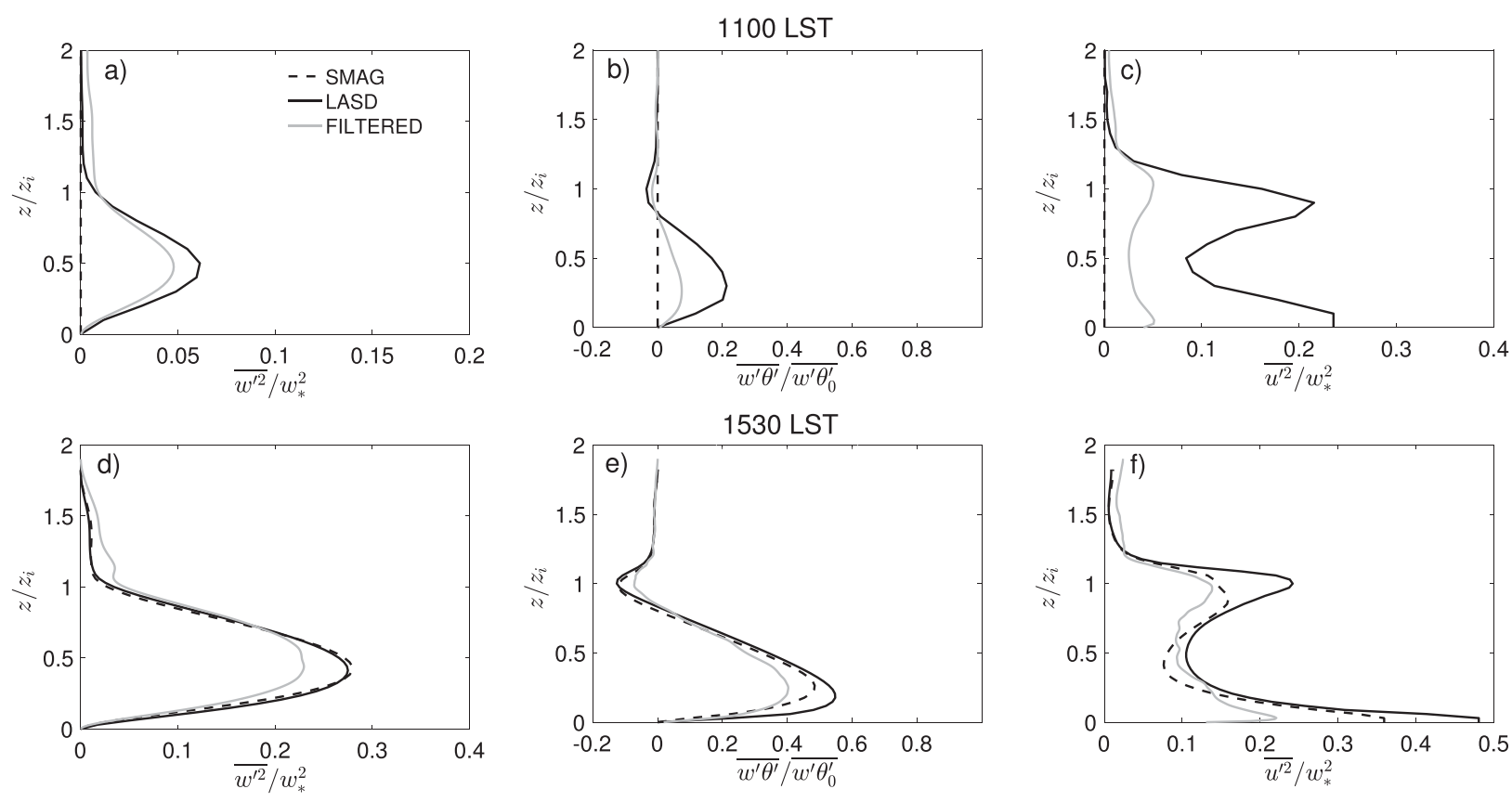

FIG. 7. As in Fig. 5, but for the 400-m simulations at 1100 and 1530 LST.

than for LASD. This difference between SMAG and LASD becomes quite marked at $\Delta x=400 \mathrm{~m}$. In that case, the LASD vertical velocity field has some small-scale features, the presence of which is not unrealistic when compared with the coarse-grained LES field, but such features are absent from the 400-m SMAG simulation. However, the smaller-scale features retained by LASD are more energetic (stronger updrafts and downdrafts) than the coarse-grained fields, which is consistent with the slightly higher TKE for LASD shown in Fig. 4.

The 2D vertical velocity power spectra from the SMAG and LASD horizontal cross sections (Fig. 8) are shown in Fig. 9. Examining the power spectra for the 100-m simulations confirms that no significant differences between the SMAG and LASD runs are present (see also Fig. 8). Dissipation, as expressed by the deviation from the inertial $k^{-5 / 3}$ spectrum, starts to affect the production scales from the 200-m simulation (Fig. 9b) becoming more pronounced at $400 \mathrm{~m}$ (Fig. 9c). Moreover, the 200- and 400-m LASD simulations seem to be slightly more energetic than SMAG near the grid scale. Nevertheless, the peak of the LASD energy spectra remains relatively unchanged close to $z_{i}$ with coarsening resolution indicating the dominant turbulence structures are well represented in LASD simulations.

\section{b. Behavior and sensitivity of $C_{S}$}

To understand the differences in behavior between the SMAG and LASD simulations across the gray zone, we need to consider the evolution of the dynamically calculated $C_{S}$ and its role in controlling the resolved turbulence. Time-height sections of the horizontally averaged $C_{S}$ for different LASD simulations are presented in Fig. 10. We note that the dynamical procedure is able to reduce $C_{S}$ close to the surface in all cases, obviating the need to apply a wall function. A strong dependence of $C_{S}$ on $\Delta x$ above the surface layer is also evident, with two different forms of behavior corresponding to the deepening and to the well-developed BL. During the erosion of the nighttime temperature inversion and deepening of the BL to around 1200 LST, $C_{S}$ is monotonically decreasing with coarsening resolution. Such behavior will be beneficial for establishing resolved turbulence in the gray-zone LASD simulations, allowing inhomogeneities to develop on the model grid more easily than in the corresponding SMAG simulations in which inhomogeneities will be more strongly diffused away. In the 400-m LASD simulation, however, the reduction in $C_{S}$ when the $\mathrm{BL}$ is shallow is somewhat larger than is desirable, leading to somewhat overenergetic turbulent structures (Figs. 4 and 7a).

When the BL becomes fully developed, $C_{S}$ is found to increase slightly with coarsening resolution until it reaches maximum values in the $\Delta x=200$-m simulation. For all runs, $C_{S}$ increases over time and achieves its maximum values near the end of the simulation, probably due to the increased instability as the BL reaches its maximum depth (Kumar et al. 2006; Basu et al. 2008). At the LES limit, those values converge to $C_{S} \approx 0.22$ in the 


\section{SMAG}
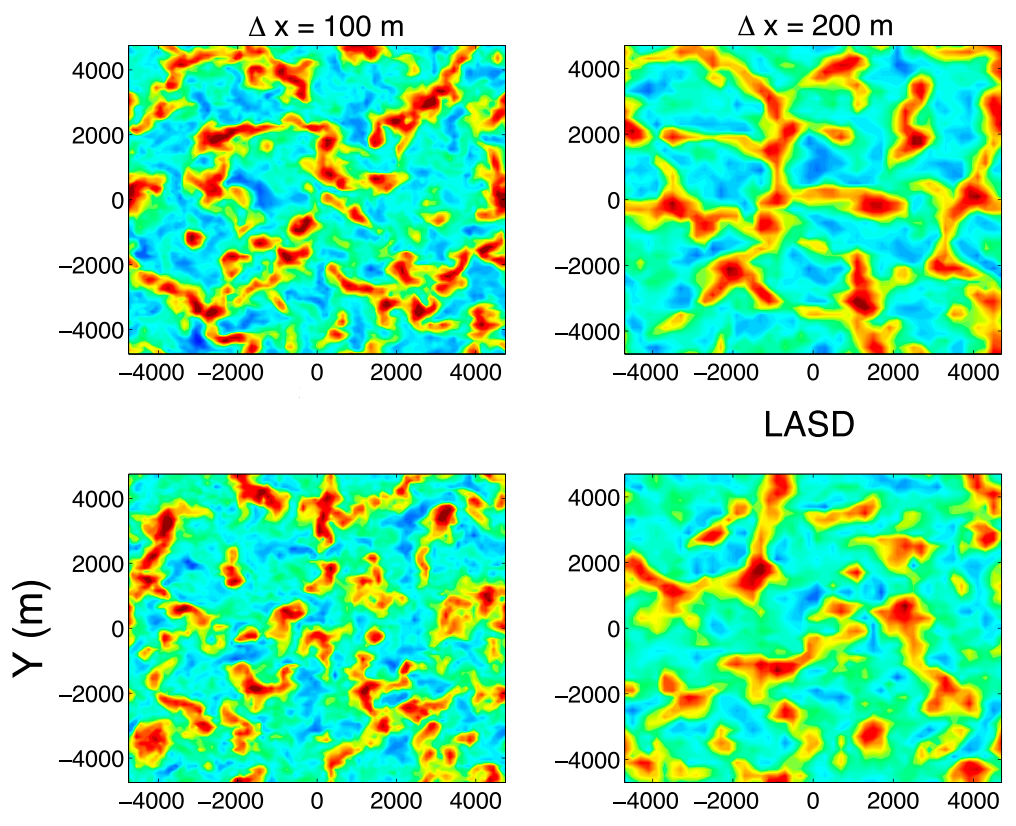

FILTERED

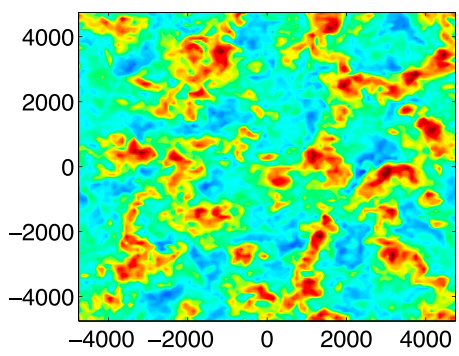

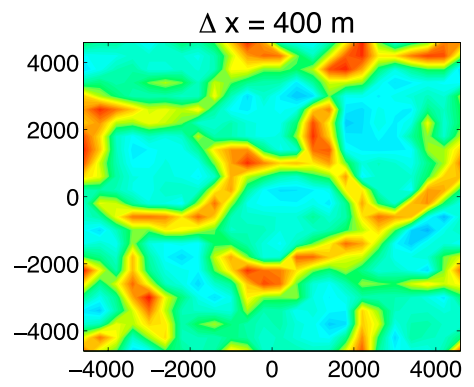
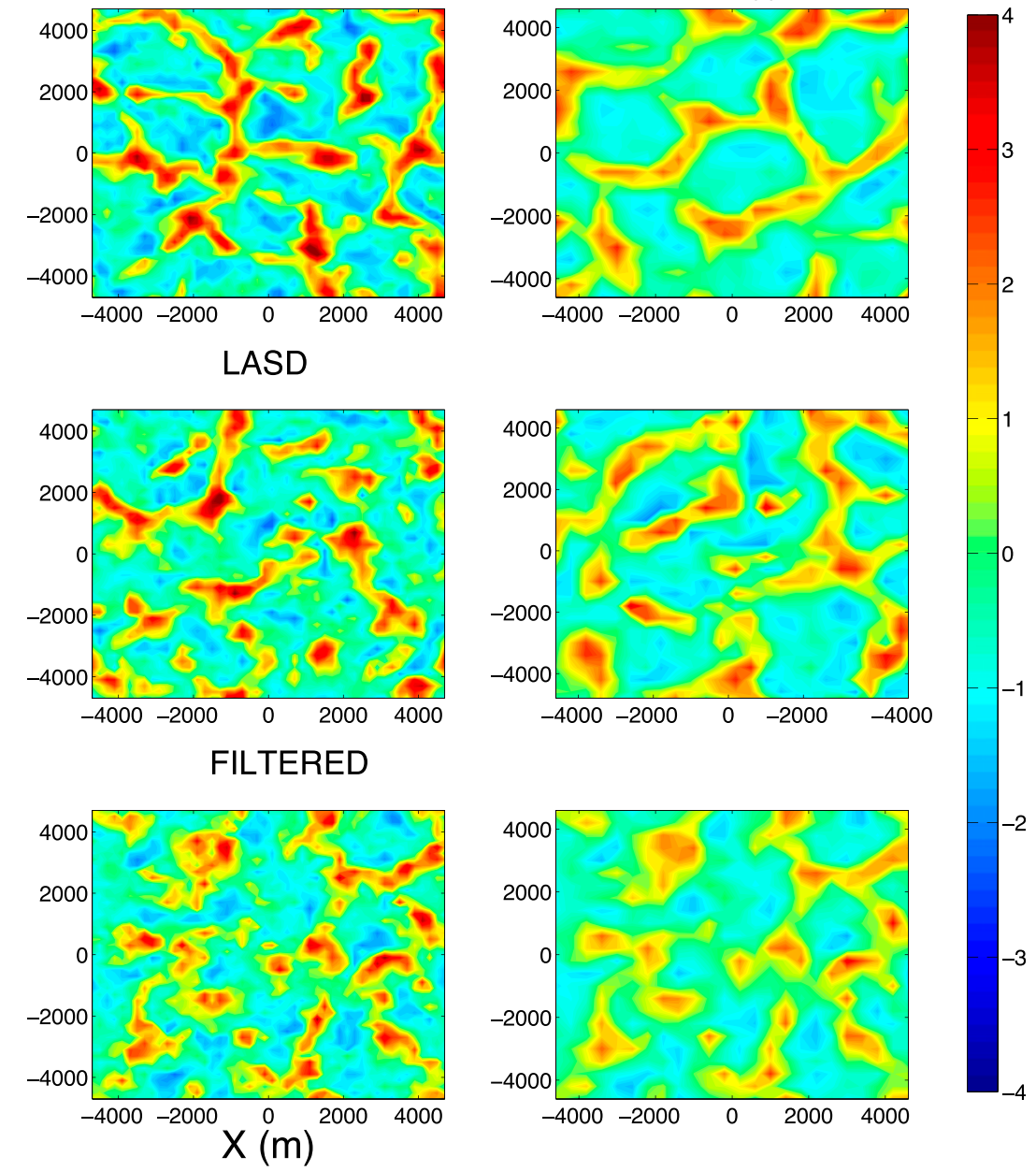

FIG. 8. Horizontal cross sections showing snapshots of the vertical velocity (color bar; $\mathrm{m} \mathrm{s}^{-1}$ ) at 1530 LST in the middle of the BL. Results are shown for the (left) 100-, (center) 200-, and (right) 400-m (top) SMAG and (middle) LASD simulations and (bottom) coarsegrained LES fields.

mixed layer, similar to the default LEM configuration. As discussed in Pope (2004), for example, the Smagorinsky coefficient should attain an appropriate equilibrium value consistent with the known inertial-range scaling law when $\Delta$ falls in the inertial subrange. However, it seems that scale dependence is still important in the evolving shallow BL even in the LES limit. In any case, reducing $C_{S}$ cannot by itself improve the representation of turbulence in the gray zone, as shown in Efstathiou and Beare (2015).

The $C_{S}$ exhibits larger values near the inversion height, particularly so in the gray-zone 200- and 400-m simulations. This occurs partly because of the increase of resolved TKE as the entrainment zone becomes underresolved (Beare 2014; Efstathiou and Beare 2015; Shin and Dudhia 2016) and partly from the impact of the stability functions on mixing [Eqs. (24) and (25)]. The determination of the $C_{S}$ values above the $\mathrm{BL}$ is controlled by the stability functions that force the subgrid fluxes to zero (see also Fig. 12).

To assess the role of scale dependence in the LASD formulation, Fig. 11a shows the vertical profile of the horizontally averaged parameter $\beta$ for different resolutions at 1530 LST. As the grid spacing is increased reaching gray-zone resolutions, $\beta$ is significantly reduced. The reduction of $\beta$ with decreasing resolution shows that the scale dependence becomes important even in the interior of the flow. This implies that the assumption of a clear inertial subrange breaks down because the dominant turbulence production scales are significantly affected by dissipation. The violation of the inertial subrange assumption becomes more obvious at 

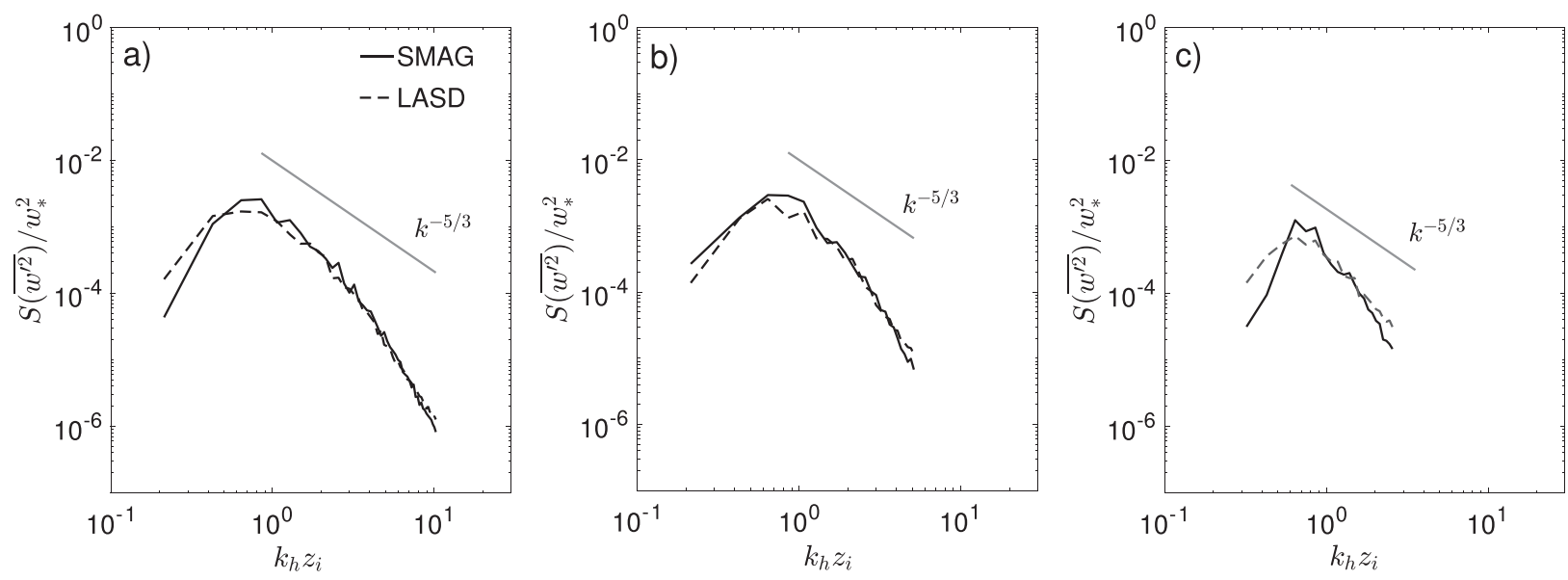

FIG. 9. The 2D vertical velocity normalized power spectra from SMAG (solid line) and LASD (dashed line) for $\Delta x=$ (a) 100, (b) 200, and (c) $400 \mathrm{~m}$ at $1530 \mathrm{LST}$ in the middle of the BL as a function of the normalized horizontal wavenumber $k_{h} z_{i}$. The $k^{-5 / 3}$ law is plotted in gray.

$400 \mathrm{~m}$, as seen from the inspection of the energy spectra in Fig. 9. Xie et al. (2015) also found a similar sensitivity of $\beta$ with grid spacing while their LES simulations of a neutral BL predicted $\beta \approx 0.6$, not dissimilar to the values obtained here.

Figure $11 \mathrm{~b}$ shows the vertical profile of the averaged $C_{S_{2 \Delta}}$ and $C_{S_{4 \Delta}}$ [see Eqs. (19) and (20)] for the 100-, 200-, and 400-m simulations at 1530 LST. For all simulations, $C_{S_{2 \Delta}}$ is reduced with coarsening resolution and $C_{S_{4 \Delta}}$ is always less than $C_{S_{2 \Delta}}$ as expected. For the 200-m simulation, $C_{S_{2 \Delta}}$ is only a little reduced relative to the same quantity in the $100-\mathrm{m}$ simulation. However, there is a significant reduction in $C_{S_{4 \Delta}}$ at $200 \mathrm{~m}$ because of the small resolved fluxes at $4 \Delta$ filter scale $(800 \mathrm{~m})$. This leads to the high $C_{S}$ values in the 200-m simulation (Fig. 10). At the 400-m simulation, $C_{S_{2 \Delta}}$ is further reduced relative to its value in the $200-\mathrm{m}$ simulation and $C_{S_{4 \Delta}}$ remains small, especially near the ground, as the method is sampling from almost unresolved scales at $1600 \mathrm{~m}$.

The formulation of the LASD in this study includes the stability impacts on the mixing length calculation through the use of stability functions. To quantify the effects of stratification on the subgrid mixing length scales, a stability mixing length $\lambda_{S}$ is introduced equal to

$$
\lambda_{S}=\lambda f_{m}(\mathrm{Ri})
$$

Figure 12 presents the time-height variation of the horizontally averaged $\lambda_{S} / \Delta$ from the SMAG and LASD $50-400-\mathrm{m}$ simulations. Subgrid mixing is confined close to the ground for the $50-\mathrm{m}$ runs for both schemes (Figs. 12a,b) with SMAG exhibiting stronger mixing on the top of the morning residual layer. For the coarser resolution simulations, SMAG significantly increases the stability mixing length relative to the grid scale when the $\mathrm{BL}$ is shallow and the simulations lie within the gray zone. The excessive damping becomes more pronounced at $200 \mathrm{~m}$, and especially in the $400-\mathrm{m}$ simulation (Figs. 12e,g), and persists through the whole morning CBL development, delaying the onset of resolved turbulence (see also Fig. 4). In contrast, LASD moderates the stability mixing length scale as a fraction of the grid scale at the same resolutions, reducing subgrid mixing (Figs. 12f,h).

To examine the impact of the numerical scheme and $\operatorname{Pr}$ on the LASD simulations, additional runs with $\Delta x=400 \mathrm{~m}$ were performed using TVD on both momentum and potential temperature and setting Pr to 0.5 and 1 . As shown in Beare (2014), TVD is more dissipative than the PiasceckWilliams scheme, and thus changing the advection scheme to TVD imposes stronger implicit numerical diffusion on the momentum field. Figure 13 presents the vertical profile of the averaged $C_{S}$ (Fig. 13a) and resolved TKE from the sensitivity simulations at 1200 LST. Changing to TVD leads to extra numerical dissipation, but LASD adapts by reducing $C_{S}$ (Fig. 13a) compared to the control (CNTL). As a result, the resolved TKE is only slightly different compared to CNTL. In a similar way, changes to the Prandtl number also induce changes to the dynamical $C_{S}$ that leave the resolved TKE profile relatively unaffected.

\section{Discussion}

The variation of $C_{S_{\Delta}}$ with horizontal resolution is dominated by the estimation of $C_{S}$ for different scales as expressed through the $\beta$ parameter. We find a decrease of $C_{S_{2 \Delta}}$ and $C_{S_{4 \Delta}}$ with increasing $\Delta x$, but the gap between the two is significantly more pronounced in the $200-\mathrm{m}$ simulation, especially when the BL is well developed. In the 200-m run, the LASD method is sampling the flow from 

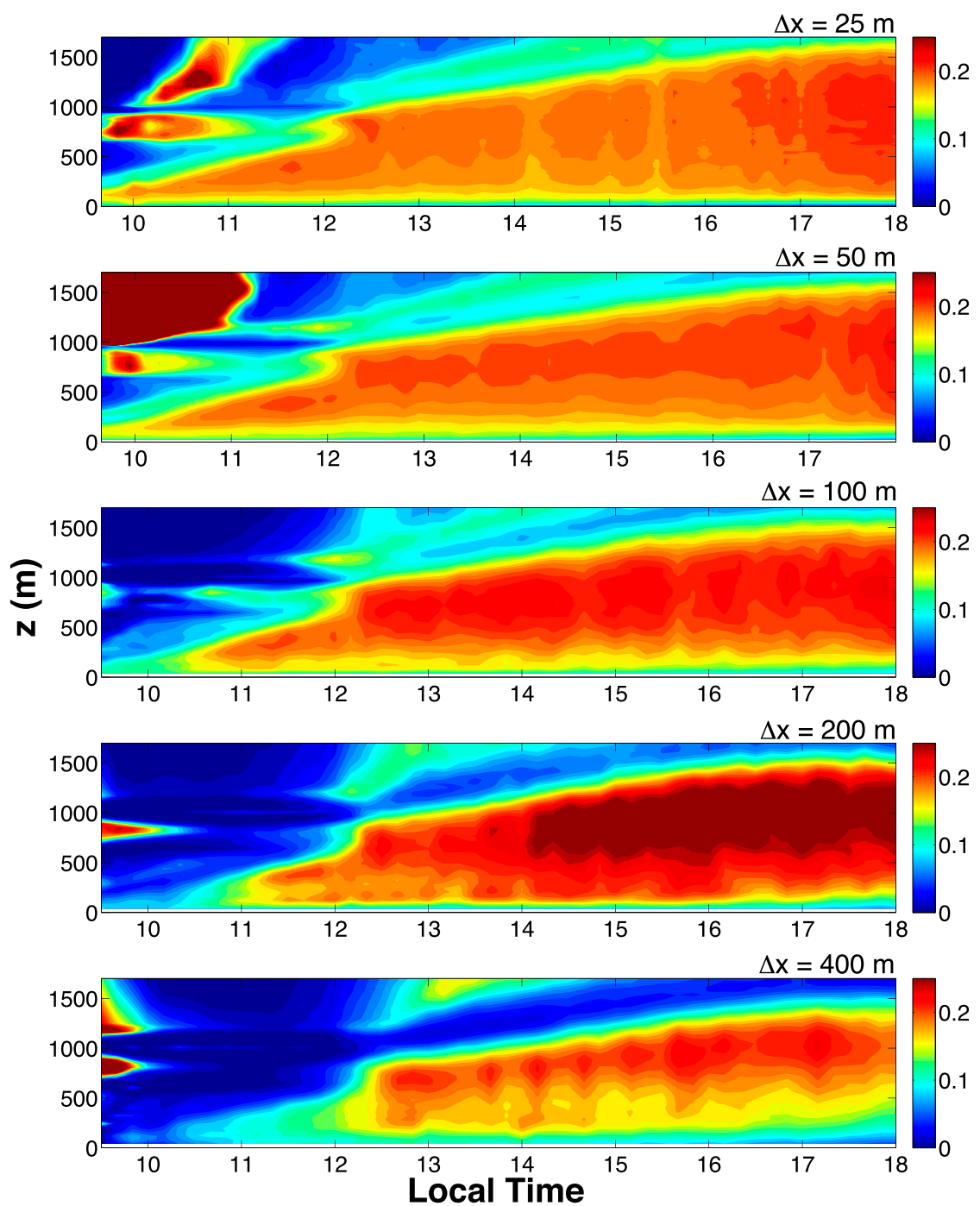

FIG. 10. Time-height sections of the horizontally averaged Smagorinsky coefficient $C_{S}$ from the LASD simulations with $\Delta x$ ranging from (top) 25 to (bottom) $400 \mathrm{~m}$.

scales of $4 \Delta=800 \mathrm{~m}$, at which little TKE is resolved, especially when the $\mathrm{BL}$ is shallow so that $z_{i} \sim \Delta x$ (Efstathiou and Beare 2015; Efstathiou et al. 2016). This leads to small $C_{S_{4 \Delta}}$ values. In the $400 \mathrm{~m}$, both the $2 \Delta$ and $4 \Delta$ filters are sampling from scales at which very little TKE is resolved, as is evident from the low $C_{S_{2 \Delta}}$ and very low $C_{S_{4 \Delta}}$ values.

The reduction of subfilter resolvable fluxes when resolution is coarse compared to the $\mathrm{BL}$ depth results in a reduction of $C_{S}$ and a decrease in the applied subgrid diffusion. This behavior can be very beneficial because it leads to much faster spinup of resolved turbulence in the LASD gray-zone simulations compared to the standard SMAG runs. It becomes obvious from Figs. 4, 6, 7, and 12 that SMAG becomes overdissipative in the gray zone that leads to enhanced dumping of the resolved fields (see also Efstathiou and Beare 2015). However, the scale-dependent model can encounter difficulties if it is required to sample from scales with very weak resolvable fluxes. Too much reduction of the values of $C_{S}$ can occur, resulting in a pileup of energy (Porté-Agel et al. 2000) and some excess of the TKE as seen in Figs. 4 and 7. There is also some evidence for such behavior near the ground, where $\overline{u^{\prime 2}}$ is large relative to the filtered LES fields for all of the LASD simulations, especially in the morning hours (Figs. 5, 6, and 7). Similar behavior was also found in Kirkil et al. (2012), where the LASD produced the greatest turbulence intensity (i.e., velocity variances) near the ground under neutral stability conditions. According to Xie et al. (2015), dynamic models do not provide sufficient dissipation compared to the truncation errors when a finite-difference discretization is used, leading to some numerical noise, even though LASD was shown to be more effective at 
a)

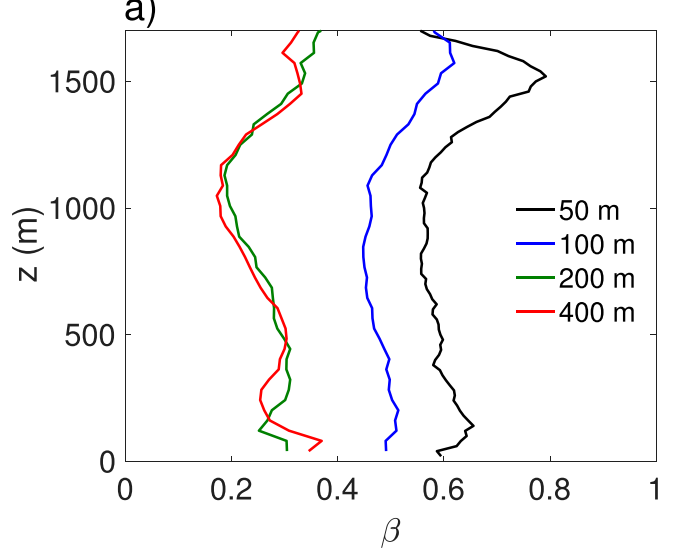

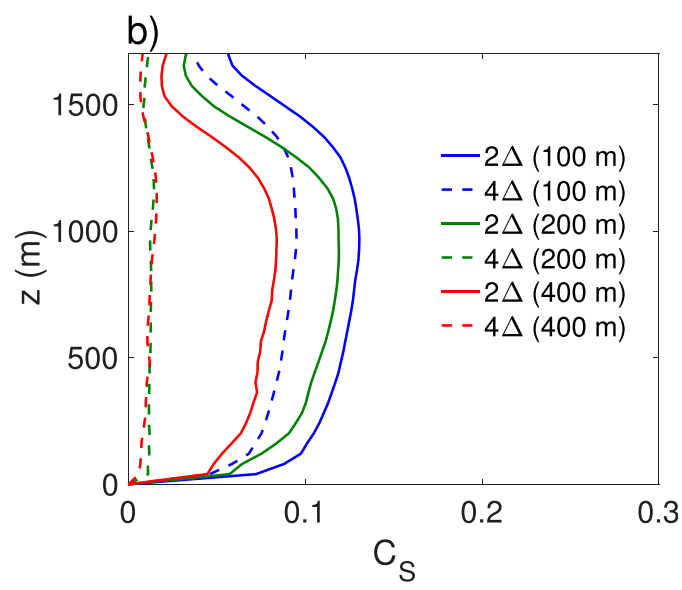

FIG. 11. Vertical profiles of horizontally averaged (a) $\beta$ parameter and (b) $C_{S_{2 \Delta}}$ (solid lines) and $C_{S_{4 \Delta}}$ (dashed lines) for different resolutions from the LASD simulations at 1530 LST. Results are shown for $\Delta x=50$ (black), 100 (blue), 200 (green), and $400 \mathrm{~m}$ (red).

removing such noise compared to other dynamic Smagorinsky models (Xie et al. 2015).

Nonetheless, as shown in Ching et al. (2014), grayzone grids are prone to developing unrealistic, strong gridscale convection. Overenergetic resolved structures can distort and mask mesoscale circulations such as local breezes (Ching et al. 2014). Efstathiou and Beare (2015) pointed out the significance of well-behaved convective overturning and used the energetics of the coarsegrained fields as a reference to quantify the amount of resolved TKE in the gray zone. Here, we find that LASD is able to reproduce the coarse-grained fields and improve the representation of turbulence compared to the standard SMAG at least for the resolutions considered in this case study. This is further supported by the examination of the spectra in Fig. 9.

The inclusion of the stability functions in the dynamic calculations (see also Kirkpatrick et al. 2006) in a selfconsistent manner, by filtering Ri through the temperature and wind fields, ensures that stability effects are taken into account in a more physical way. However, this approach is different than in most dynamic models where the effects of stability are explicitly represented by $C_{S}$. Nevertheless, the use of the stability functions has no significant impact on the LASD results at the LES limit (not shown) as the dominant turbulence eddies are resolved, whereas their effect becomes more pronounced in the gray zone. The impact of stability functions on the dynamic calculations is an issue that deserves further investigation.

Within the current LEM configuration, the $C_{S_{\Delta}}$ tendency toward zero marks a usability limit for the LASD. We have found the dynamic reductions in $C_{S_{\Delta}}$ to be genuinely useful within the gray-zone regime, but if $C_{S_{\Delta}}$ becomes too small then the subgrid model ultimately becomes ineffective at exerting control over the flow properties near the grid scale, which are then determined only by the model numerics. Extending the present set of simulations to $\Delta x=800 \mathrm{~m}$ makes the LEM numerically unstable as a result of the lack of sufficient subgrid mixing (not shown). It should be remarked that using less dissipative forms of the stability functions did not change the results in a qualitative sense and did not have an impact on the usability limit of the LASD. This illustrates that there are limitations on the formulation of the closure model itself, and a Smagorinsky-based scheme might not be able to reproduce the transition of the subgrid fluxes in the entire gray zone, making it more appropriate for use in the near gray zone.

Wyngaard (2004) showed that eddy viscosity and diffusivity should be considered tensors rather than as scalars, at least in the gray zone. Even though the LASD is formulated on the basis of a simple scalar eddy-viscosity approach, the dynamically derived mixing length depends on the 3D wind shear and resolved momentum fluxes at different scales, and it includes information about the flow history through the Lagrangian averaging procedure. These features seem to match some of the benefits that Wyngaard (2004) identifies in a tensor approach. A scale-dependent mixing length is in accordance with the unified closure concept for the dominant turbulence length scales as suggested by Wyngaard (2004).

\section{Conclusions}

A scale-dependent Lagrangian-averaged model for the dynamic calculation of $C_{S}$ in the Smagorinsky subgrid model was applied in the LEM to simulate an evolving convective $\mathrm{BL}$ at a range of horizontal resolutions, ranging from LES to the gray zone. The LASD was 

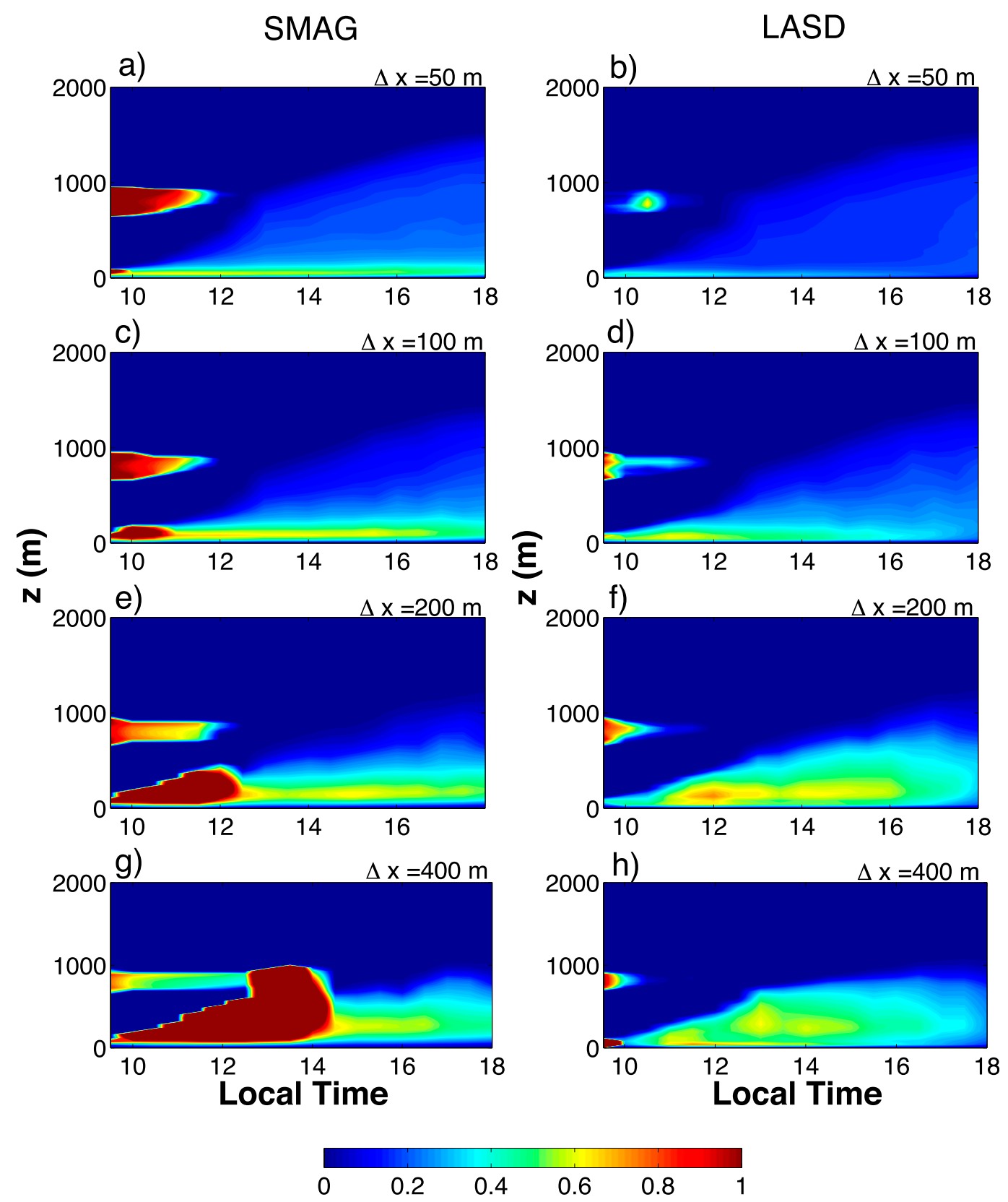

FIG. 12. Time-height variation of the horizontally averaged $\lambda_{S} / \Delta$ from the (left) SMAG and (right) LASD (a), (b) 50-, (c),(d) 100-, (e),(f) 200-, and (g),(h) 400-m simulations.

modified to account for the stratification effects in the atmosphere by including the stability functions in the dynamic calculation.

Results from the LASD simulations exhibit a relative insensitivity to resolution compared to SMAG as shown by Basu et al. (2008), adjusting the value of $C_{S}$ according to the characteristics of the resolved flow. Besides its scale dependency, LASD also shows increased adaptability to the imposed dissipation from the advection scheme and other model parameters. The dynamic model has some clear advantages over SMAG in reproducing the first- and second-order profiles across the scales. It is able to reproduce second-order turbulence statistics from the filtered LES fields even when coarser resolution is used. The use of the LASD can add up to a factor of 2 in computational time relative to SMAG. However, note that the LEM is parallelized on 1D slices, which significantly limits its scalability. Moreover, no special care has been taken 

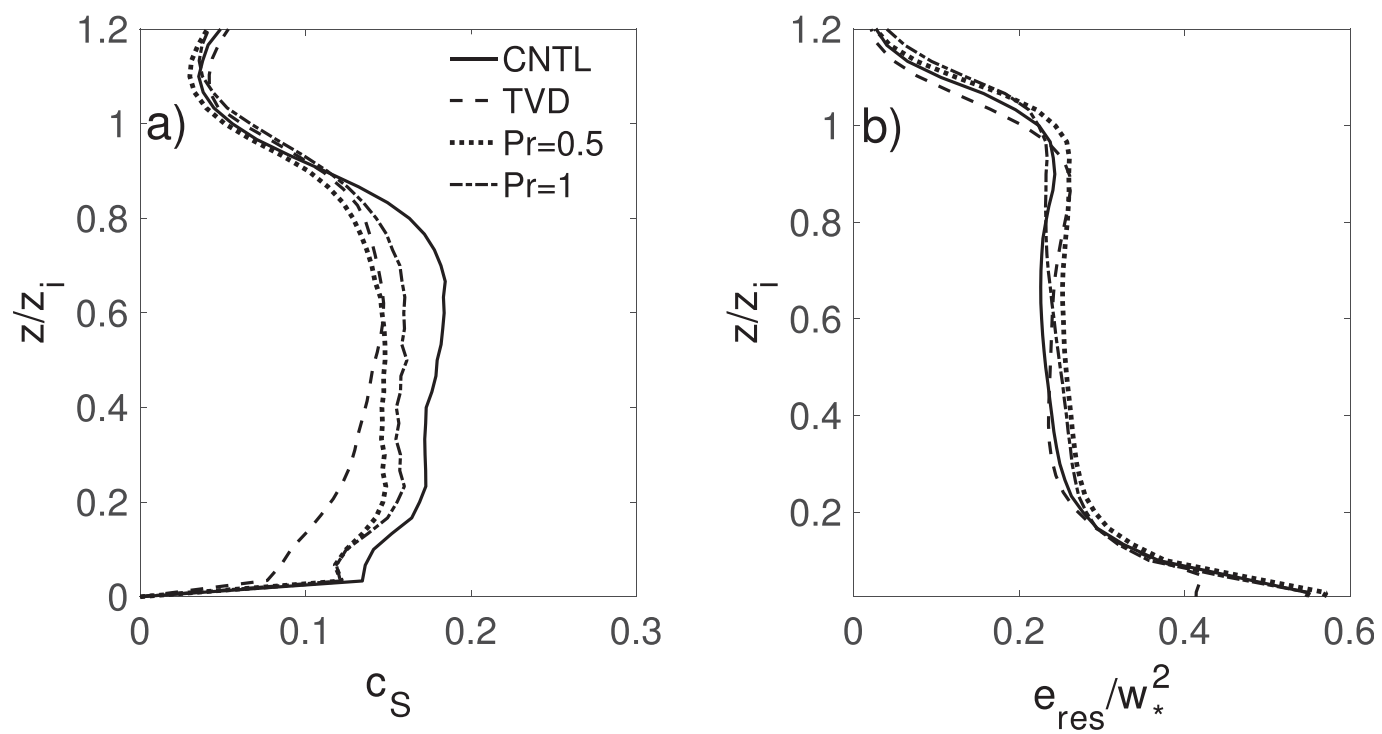

FIG. 13. Vertical profiles of horizontally averaged (a) $C_{S}$ and (b) normalized resolved TKE for the different sensitivity runs using LASD with $\Delta x=400 \mathrm{~m}$ at $1200 \mathrm{LST}$. Results are shown for the control run (solid lines) and for the TVD with $\operatorname{Pr}=0.7$ (as control; dashed lines), 0.5 (dotted), and 1.0 (dash-dotted).

toward optimizing the LASD code for its use in this paper because running the LEM in the near gray zone is relatively cheap. The main deficiency of the LASD is that it is somewhat overenergetic, especially near the surface, which may be attributable to the finite-difference discretization of the LEM. This becomes more pronounced as the resolution becomes coarser and $C_{S}$ values tend toward zero. However, first-order quantities (potential temperature profiles) are relatively unaffected, remaining close to the LES profiles at all of the tested resolutions.

The dynamically derived $C_{S}$ displays a clear dependence on grid spacing. The variation of the $C_{S}$ calculation with scale at the filter scales $2 \Delta$ and $4 \Delta$ plays an important role in those dependencies. During the shallow stage of morning CBL development, $C_{S}$ is reduced with coarsening resolution because the dynamic procedure is sampling from almost unresolved scales as $\Delta x / z_{i}$ increases. This has the beneficial effect of allowing a realistic level of turbulence to become established earlier on a gray-zone grid. When the BL has developed, $C_{S}$ is found to increase as the resolution coarsens for the reason that $C_{S_{44}}$ decreases more rapidly in the gray zone than does $C_{S_{2 \Delta}}$. For the LASD simulation with $\Delta x=$ $400 \mathrm{~m}$, the value of $C_{S}$ is reduced somewhat in comparison with the case of $\Delta x=200 \mathrm{~m}$. The LASD starts to approach a usability limit, for the case examined and the current LEM configuration, when $\Delta$ becomes sufficiently large that $C_{S_{4 \Delta}}$ is too small. Nonetheless, this study has shown that the scale-dependent model is able to improve the simulation of an evolving CBL at resolutions that are already achievable by regional NWP models (Hanley et al. 2015). It may be used as an alternative to the standard Smagorinsky approach at least in the near gray zone: that is, the regime between the strictly converging LES (Sullivan and Patton 2011) and the defined gray zone (Beare 2014). However, because the Wangara day-33 experiment is a buoyancy-driven case study, with the Monin-Obukov length $L$ staying within $-1>L>-5 \mathrm{~m}$ for most of the daytime (Deardorff 1974), the effects of strong wind shear are not taken into account, and therefore this study is limited to the free-convection regime. Further work is currently under way to assess whether the advantages of a dynamic model might be brought to bear at even coarser resolutions by blending LASD with a 1D nonlocal BL scheme in a similar fashion to the pragmatic blending scheme of Boutle et al. (2014).

Acknowledgments. This work has been funded by the Natural Environment Research Council (NERC) GREYBLS (Modelling Grey Zone Boundary Layers) project (University of Exeter component Grant NE/ K011456/1; University of Reading component Grant NE/ K011502/1). We acknowledge the use of the MONSooN system, a collaborative facility supplied under the Joint Weather and Climate Research Programme, which is a strategic partnership between the Met Office and the Natural Environment Research Council. The authors thank the three anonymous reviewers for their comments and valuable suggestions, which helped to improve this paper. The research materials supporting this publication can be accessed by contacting the corresponding author. 


\section{REFERENCES}

Basu, S., and F. Porté-Agel, 2006: Large-eddy simulation of stably stratified atmospheric boundary layer turbulence: A scaledependent dynamic modeling approach. J. Atmos. Sci., 63, 2074-2091, https://doi.org/10.1175/JAS3734.1.

_ - J.-F. Vinuesa, and A. Swift, 2008: Dynamic LES modeling of a diurnal cycle. J. Appl. Meteor. Climatol., 47, 1156-1174, https://doi.org/10.1175/2007JAMC1677.1.

Beare, R. J., 2014: A length scale defining partially-resolved boundary-layer turbulence simulations. Bound.-Layer Meteor., 151, 39-55, https://doi.org/10.1007/s10546-013-9881-3.

Bhattacharya, R., and B. Stevens, 2016: A two turbulence kinetic energy model as a scale-adaptive approach to modeling the planetary boundary layer. J. Adv. Model. Earth Syst., 8, 224 243, https://doi.org/10.1002/2015MS000548.

Bou-Zeid, E., C. Meneveau, and M. Parlange, 2005: A scaledependent Lagrangian dynamic model for large eddy simulation of complex turbulent flows. Phys. Fluids, 17, 025105, https://doi.org/10.1063/1.1839152.

Boutle, I. A., J. E. J. Eyre, and A. P. Lock, 2014: Seamless stratocumulus simulation across the turbulent gray zone. Mon. Wea. Rev., 142, 1655-1668, https://doi.org/10.1175/ MWR-D-13-00229.1.

Brown, A. R., S. H. Derbyshire, and P. J. Mason, 1994: Large-eddy simulation of stable atmospheric boundary layers with a revised stochastic subgrid model. Quart. J. Roy. Meteor. Soc., 120, 1485-1512, https://doi.org/10.1002/qj.49712052004.

Ching, J., R. Rotunno, M. LeMone, A. Martilli, B. Kosovic, P. A. Jimenez, and J. Dudhia, 2014: Convectively induced secondary circulations in fine-grid mesoscale numerical weather prediction models. Mon. Wea. Rev., 142, 3284-3302, https:// doi.org/10.1175/MWR-D-13-00318.1.

Clarke, R. H., A. J. Dyer, R. R. Brook, D. G. Reid, and A. J. Troup, 1971: The Wangara experiment: Boundary layer data. CSIRO Tech. Rep. 19, 358 pp.

Deardorff, J. W., 1974: Three-dimensional numerical study of turbulence in an entraining mixed layer. Bound.-Layer Meteor., 7, 199-226, https://doi.org/10.1007/BF00227913.

Efstathiou, G. A., and R. J. Beare, 2015: Quantifying and improving sub-grid diffusion in the boundary-layer grey zone Quart. J. Roy. Meteor. Soc., 141, 3006-3017, https://doi.org/ 10.1002/qj.2585.

,,-- S. Osborne, and A. P. Lock, 2016: Grey zone simulations of the morning convective boundary layer development. J. Geophys. Res. Atmos., 121, 4769-4782, https://doi.org/ 10.1002/2016JD024860.

Germano, M., U. Piomelli, P. Moin, and W. H. Cabot, 1991: A dynamic subgrid-scale eddy viscosity model. Phys. Fluids, 3A, 1760-1765, https://doi.org/10.1063/1.857955.

Glendening, J. W., and T. Haack, 2001: Influence of advection differencing error upon large-eddy simulation accuracy. Bound.-Layer Meteor., 98, 127-153, https://doi.org/10.1023/ A:1018734205850.

Hanley, K. E., R. S. Plant, T. H. M. Stein, R. J. Hogan, J. C. Nicol, H. W. Lean, C. Halliwell, and P. A. Clark, 2015: Mixing-length controls on high-resolution simulations of convective storms. Quart. J. Roy. Meteor. Soc., 141, 272-284, https://doi.org/ 10.1002/qj.2356.

Hong, S.-Y., Y. Noh, and J. Dudhia, 2006: A new vertical diffusion package with an explicit treatment of entrainment processes. Mon. Wea. Rev., 134, 2318-2341, https://doi.org/10.1175/ MWR3199.1.
Honnert, R., V. Masson, and F. Couvreux, 2011: A diagnostic for evaluating the representation of turbulence in atmospheric models at the kilometric scale. J. Atmos. Sci., 68, 3112-3131, https://doi.org/10.1175/JAS-D-11-061.1.

, F. Couvreux, V. Masson, and D. Lancz, 2016: Sampling the structure of convective turbulence and implications for greyzone parametrizations. Bound.-Layer Meteor., 160, 133-156, https://doi.org/10.1007/s10546-016-0130-4.

Huang, H.-Y., B. Stevens, and S. A. Margulis, 2008: Application of dynamic subgrid-scale models for large-eddy simulation of the daytime convective boundary layer over heterogeneous surfaces. Bound.-Layer Meteor., 126, 327-348, https://doi.org/ 10.1007/s10546-007-9239-9.

Ito, J., H. Niino, M. Nakanishi, and C.-H. Moeng, 2015: An extension of the Mellor-Yamada model to the terra incognita zone for dry convective mixed layers in the free convection regime. Bound.-Layer Meteor., 157, 23-43, https://doi.org/ 10.1007/s10546-015-0045-5.

Kirkil, G., J. Mirocha, E. Bou-Zeid, F. K. Chow, and B. Kosović, 2012: Implementation and evaluation of dynamic subfilterscale stress models for large-eddy simulation using WRF. Mon. Wea. Rev., 140, 266-284, https://doi.org/10.1175/ MWR-D-11-00037.1.

Kirkpatrick, M. P., A. S. Ackerman, D. E. Stevens, and N. N. Mansour, 2006: On the application of the dynamic Smagorinsky model to large-eddy simulations of the cloud-topped atmospheric boundary layer. J. Atmos. Sci., 63, 526-546, https:// doi.org/10.1175/JAS3651.1.

Kober, K., and G. C. Craig, 2016: Physically based stochastic perturbations (PSP) in the boundary layer to represent uncertainty in convective initiation. J. Atmos. Sci., 73, 2893-2911, https://doi.org/10.1175/JAS-D-15-0144.1.

Kumar, V., J. Kleissl, C. Meneveau, and M. B. Parlange, 2006: Largeeddy simulation of a diurnal cycle of the atmospheric boundary layer: Atmospheric stability and scaling issues. Water Resour. Res., 42, W06D09, https://doi.org/10.1029/2005WR004651.

Leonard, B. P., M. K. Macvean, and A. P. Lock, 1993: Positivitypreserving numerical schemes for multidimensional advection. NASA Tech. Memo. NASA-TM-106055, 62 pp.

Leoncini, G., R. S. Plant, S. L. Gray, and P. A. Clark, 2013: Ensemble forecasts of a flood-producing storm: Comparison of the influence of model-state perturbations and parameter modifications. Quart. J. Roy. Meteor. Soc., 139, 198-211, https://doi.org/10.1002/qj.1951.

Lilly, D. K., 1967: The representation of small-scale turbulence in numerical simulation experiments. Proc. IBM Scientific Computing Symp. on Environmental Sciences, Yorktown Heights, NY, IBM, 195-210.

_ 1992: A proposed modification of the Germano subgrid-scale closure method. Phys. Fluids, 4A, 633-635, https://doi.org/ 10.1063/1.858280.

Lock, A. P., A. R. Brown, M. R. Bush, G. M. Martin, and R. N. B. Smith, 2000: A new boundary layer mixing scheme. Part I: Scheme description and single-column model tests. Mon. Wea. Rev., 128, 3187-3199, https://doi.org/10.1175/1520-0493(2000) $128<3187$ :ANBLMS > 2.0.CO;2; Corrigendum, 129, 905, https://doi.org/10.1175/1520-0493(2001)129<0905:C >2.0.CO;2.

Mason, P. J., and D. J. Thomson, 1992: Stochastic backscatter in large-eddy simulations of boundary layers. J. Fluid Mech., 242, 51-78, https://doi.org/10.1017/S0022112092002271.

Meneveau, C., T. S. Lund, and W. H. Cabot, 1996: A Lagrangian dynamic subgrid-scale model of turbulence. J. Fluid Mech., 319, 353-385, https://doi.org/10.1017/S0022112096007379. 
Nakanishi, M., R. Shibuya, J. Ito, and H. Niino, 2014: Large-eddy simulation of a residual layer: Low-level jet, convective rolls, and Kelvin-Helmholtz instability. J. Atmos. Sci., 71, 44734491, https://doi.org/10.1175/JAS-D-13-0402.1.

Piacsek, S. A., and G. P. Williams, 1970: Conservation properties of convection difference schemes. J. Comput. Phys., 6, 392-405, https://doi.org/10.1016/0021-9991(70)90038-0.

Pope, S. B., 2004: Ten questions concerning the large-eddy simulation of turbulent flows. New J. Phys., 6, 35, https://doi.org/ 10.1088/1367-2630/6/1/035.

Porté-Agel, F., C. Meneveau, and M. B. Parlange, 2000: A scaledependent dynamic model for large-eddy simulation: Application to a neutral atmospheric boundary layer. J. Fluid Mech., 415, 261-284, https://doi.org/10.1017/S0022112000008776.

Shin, H. H., and S.-Y. Hong, 2013: Analysis of resolved and parameterized vertical transports in convective boundary layers at gray-zone resolutions. J. Atmos. Sci., 70, 3248-3261, https:// doi.org/10.1175/JAS-D-12-0290.1.

- and — 2015: Representation of the subgrid-scale turbulent transport in convective boundary layers at gray-zone resolutions. Mon. Wea. Rev., 143, 250-271, https://doi.org/10.1175/ MWR-D-14-00116.1.

, and J. Dudhia, 2016: Evaluation of PBL parameterizations in WRF at subkilometer grid spacings: Turbulence statistics in the dry convective boundary layer. Mon. Wea. Rev., 144, 11611177, https://doi.org/10.1175/MWR-D-15-0208.1.

Smagorinsky, J., 1963: General circulation experiments with the primitive equations. Mon. Wea. Rev., 91, 99-164, https://doi.org/ 10.1175/1520-0493(1963)091<0099:GCEWTP>2.3.CO;2.
Stein, T. H. M., R. J. Hogan, P. A. Clark, C. E. Halliwell, K. E. Hanley, H. W. Lean, J. C. Nicol, and R. S. Plant, 2015: The DYMECS project: A statistical approach for the evaluation of convective storms in high-resolution NWP models. Bull. Amer. Meteor. Soc., 96, 939-951, https://doi.org/10.1175/ BAMS-D-13-00279.1.

Stoll, R., and F. Porté-Agel, 2008: Large-eddy simulation of the stable atmospheric boundary layer using dynamic models with different averaging schemes. Bound.-Layer Meteor., 126, 1-28, https://doi.org/10.1007/s10546-007-9207-4.

Sullivan, P. P., and E. G. Patton, 2011: The effect of mesh resolution on convective boundary layer statistics and structures generated by large-eddy simulation. J. Atmos. Sci., 68, 2395-2415, https://doi. org/10.1175/JAS-D-10-05010.1; Corrigendum, 69, 1155, https:// doi.org/10.1175/JAS-D-11-0325.1.

Thurston, W., R. J. B. Fawcett, K. J. Tory, and J. D. Kepert, 2016: Simulating boundary-layer rolls with a numerical weather prediction model. Quart. J. Roy. Meteor. Soc., 142, 211-223, https://doi.org/10.1002/qj.2646.

Wyngaard, J. C., 2004: Toward numerical modeling in the "terra incognita.' J. Atmos. Sci., 61, 1816-1826, https://doi.org/10.1175/ 1520-0469(2004)061<1816:TNMITT>2.0.CO;2.

Xie, S., N. Ghaisas, and C. L. Archer, 2015: Sensitivity issues in finitedifference large-eddy simulations of the atmospheric boundary layer with dynamic subgrid-scale models. Bound-Layer Meteor., 157, 421-445, https://doi.org/10.1007/s10546-015-0071-3.

Zhou, B., J. S. Simon, and F. K. Chow, 2014: The convective boundary layer in the terra incognita. J. Atmos. Sci., 71, 25452563, https://doi.org/10.1175/JAS-D-13-0356.1. 